ALEA, Lat. Am. J. Probab. Math. Stat. 15, 1335-1375 (2018)

DOI: $10.30757 /$ ALEA.v15-50

\title{
Transition density estimates for diagonal systems of SDEs driven by cylindrical $\alpha$-stable processes
}

\section{Tadeusz Kulczycki and Michał Ryznar}

Faculty of Pure and Applied Mathematics, Wrocław University of Science and Technology Wyb. Wyspiańskiego 27, 50-370 Wrocław, Poland.

E-mail address: Tadeusz.Kulczycki@pwr.edu.pl

Faculty of Pure and Applied Mathematics, Wrocław University of Science and Technology Wyb. Wyspiańskiego 27, 50-370 Wrocław, Poland.

E-mail address: Michal.Ryznar@pwr.edu.pl

Abstract. We consider the system of stochastic differential equation $d X_{t}=A\left(X_{t-}\right) d Z_{t}, X_{0}=x$, driven by cylindrical $\alpha$-stable process $Z_{t}$ in $\mathbb{R}^{d}$. We assume that $A(x)=\left(a_{i j}(x)\right)$ is diagonal and $a_{i i}(x)$ are bounded away from zero, from infinity and Hölder continuous. We construct transition density $p^{A}(t, x, y)$ of the process $X_{t}$ and show sharp two-sided estimates of this density. We also prove Hölder and gradient estimates of $x \rightarrow p^{A}(t, x, y)$. Our approach is based on the method developed in Chen and Zhang (2016).

\section{Introduction}

Let

$$
Z_{t}=\left(Z_{t}^{(1)}, \ldots, Z_{t}^{(d)}\right)
$$

be cylindrical $\alpha$-stable process, that is $Z_{t}^{(i)}, i=1, \ldots, d$ are independent onedimensional symmetric standard $\alpha$-stable processes of index $\alpha \in(0,2), d \in \mathbb{N}$, $d \geq 2$. We consider the system of stochastic differential equation

$$
d X_{t}=A\left(X_{t-}\right) d Z_{t}, \quad X_{0}=x,
$$

where $A(x)=\left(a_{i j}(x)\right)$ is diagonal and there are constants $b_{1}, b_{2}, b_{3}>0, \beta \in(0,1]$ such that for any $x, y \in \mathbb{R}^{d}, i \in\{1, \ldots, d\}$

$$
b_{1} \leq a_{i i}(x) \leq b_{2},
$$

Received by the editors January 8th, 2018; accepted October 7th, 2018.

2010 Mathematics Subject Classification. 60H10.

Key words and phrases. Stochastic differential equation, stable process, cylindrical process, Lévi's method, transition density.

T. Kulczycki was supported in part by the National Science Centre, Poland, grant no. 2015/17/B/ST1/01233, M. Ryznar was supported in part by the National Science Centre, Poland, grant no. 2015/17/B/ST1/01043. 


$$
\left|a_{i i}(x)-a_{i i}(y)\right| \leq b_{3}|x-y|^{\beta} .
$$

In the sequel, without loss of generality, we assume that $\beta \in(0, \alpha / 4]$.

We define an operator $\mathcal{L}$ by the following formula

$\mathcal{L} f(x)=\sum_{i=1}^{d} \lim _{\varepsilon \rightarrow 0^{+}} \frac{\mathcal{A}_{\alpha}}{2} \int_{\left|w_{i}\right|>\varepsilon}\left[f\left(x+a_{i i}(x) w_{i} e_{i}\right)+f\left(x-a_{i i}(x) w_{i} e_{i}\right)-2 f(x)\right] \frac{d w_{i}}{\left|w_{i}\right|^{1+\alpha}}$,

for a Borel function $f$ such that the limit on the right hand side exists for any $x \in \mathbb{R}^{d}$. Here $\left\{e_{j}\right\}_{j=1}^{d}$ is the standard basis in $\mathbb{R}^{d}$ and $\mathcal{A}_{\alpha}=\frac{2^{\alpha} \Gamma((1+\alpha) / 2)}{\pi^{1 / 2}|\Gamma(-\alpha / 2)|}$.

It is well known that system of SDEs (1.1) has a unique weak solution and the generator of $X$, restricted to $C_{b}^{2}\left(\mathbb{R}^{d}\right)$, is given by $\mathcal{L}$ (see Bass and Chen, 2006).

Let us denote the transition density of one-dimensional symmetric standard $\alpha$ stable process of index $\alpha \in(0,2)$ by $g_{t}(x-y), t>0, x, y \in \mathbb{R}$. Clearly, the transition density of $Z(t)$ is given by $\prod_{j=1}^{d} g_{t}\left(x_{j}-y_{j}\right)$.

The main result of this paper is the following theorem.

Theorem 1.1. (i) The strong Markov process $X(t)$ formed by the unique weak solution to $S D E$ (1.1) has a positive jointly continuous transition density function $p^{A}(t, x, y)$ in $(t, x, y) \in(0, \infty) \times \mathbb{R}^{d} \times \mathbb{R}^{d}$ with respect to the Lebesgue measure on $\mathbb{R}^{d}$.

(ii) The transition density solves

$$
\frac{\partial}{\partial t} p^{A}(t, x, y)=\mathcal{L} p^{A}(t, \cdot, y)(x)
$$

for all $t \in(0, \infty)$ and $x, y \in \mathbb{R}^{d}$.

(iii) For any $T>0$ there exist $c_{1}=c_{1}\left(T, d, \alpha, b_{1}, b_{2}, b_{3}, \beta\right) \geq 1$ such that for any $x, y \in \mathbb{R}^{d}, t \in(0, T]$

$$
c_{1}^{-1} \prod_{i=1}^{d} g_{t}\left(x_{i}-y_{i}\right) \leq p^{A}(t, x, y) \leq c_{1} \prod_{i=1}^{d} g_{t}\left(x_{i}-y_{i}\right) .
$$

(iv) For any $T>0$ and $\gamma \in(0, \alpha \wedge 1)$ there exists $c_{2}=c_{2}\left(T, \gamma, d, \alpha, b_{1}, b_{2}, b_{3}, \beta\right)>$ 0 such that for any $x, x^{\prime}, y \in \mathbb{R}^{d}, t \in(0, T]$

$$
\left|p^{A}(t, x, y)-p^{A}\left(t, x^{\prime}, y\right)\right| \leq c_{2}\left|x-x^{\prime}\right|^{\gamma} t^{-\gamma / \alpha}\left(\prod_{i=1}^{d} g_{t}\left(x_{i}-y_{i}\right)+\prod_{i=1}^{d} g_{t}\left(x_{i}^{\prime}-y_{i}\right)\right) .
$$

(v) For any $T>0$ and $\alpha \in(1,2)$ there exist $c_{3}=c_{3}\left(T, d, \alpha, b_{1}, b_{2}, b_{3}, \beta\right)>0$ such that for any $x, y \in \mathbb{R}^{d}, t \in(0, T]$

$$
\left|\nabla_{x} p^{A}(t, x, y)\right| \leq c_{3} t^{-1 / \alpha} p^{A}(t, x, y) .
$$

Systems of stochastic differential equations driven by cylindrical $\alpha$-stable processes have attracted a lot of attention in recent years see e.g. Bass and Chen (2006); Priola and Zabczyk (2011); Wang and Zhang (2015); Priola et al. (2012); Zhang (2013); Sun and Xie (2014). In Bass and Chen (2006) existence and uniqueness of weak solutions of systems of SDEs (1.1) is proved under very mild assumptions on matrices $A(x)$ (i.e. it is assumed that $A(x)$ are continuous and bounded in $x$ and nondegenerate for each $x$ ). Our paper may be treated as the first step in studying fine properties of transition densities of systems of SDEs driven by Lévy processes with singular Lévy measures. Fine properties of such transition densities are of great interest but in the case of singular Lévy measures relatively little is 
known. We decided to study the particular case of diagonal matrices $A(x)$ in (1.1) because in that case one can obtain sharp two-sided estimates of these densities. Note that upper and lower bounds of transition densities are possible due to the specific structure of the generator, in particular, that each one dimensional measure in the representation of $\mathcal{L}$ is absolutely continuous. It seems that in the case of general non-diagonal matrices in (1.1) such sharp two-sided estimates are impossible to obtain. Nevertheless, we believe that our results will help to obtain qualitative estimates of transition densities also in the case of general matrices in (1.1).

The direct inspiration to study transition densities of solutions to (1.1) was a question of Zabczyk concerning gradient estimates of these densities. Another source of inspiration was a recent paper Bogdan et al. (2017), where the authors constructed heat kernels and obtained upper bounds and Hölder estimates of them for quite general anisotropic space-inhomogeneous non-local operators. However, the considered jump kernels cannot be "too singular". In particular, their results can be applied for systems (1.1) only when $d=2$ and $\alpha \in(1,2)$ (see the condition $\alpha+\gamma>d$ in the assumption A1 on page 5 in Bogdan et al., 2017). Moreover, even for $d=2$ and $\alpha \in(1,2)$, the obtained estimates are far from being optimal.

In our paper we use a very elegant and efficient method based on Levi's freezing coefficient argument (cf. Levi, 1907; Friedman, 1964; Ladyženskaja et al., 1968). This method was applied by Kochubei in the framework of pseudodifferential operators (see Kochuber, 1988) to construct a fundamental solution to the related Cauchy problem as well as transition density for the corresponding Markow process. Then in the work Chen and Zhang (2016) this approach was further extended to provide sharp two-sided estimates of the transition density in the case of the non-local and non-symmetric Lévy type operators on $\mathbb{R}^{d}$. Chen and Zhang studied jump kernels of the type $\kappa(x, z) /|z|^{d+\alpha}, \alpha \in(0,2)$, with some regularity conditions of $\kappa(x, z)$. It turned out that similar ideas can be applied also in our situation where jump kernels are much more singular. We follow the road-map from Chen and Zhang (2016) however, due to a specific structure of the operator $\mathcal{L}$, there are many differences between that paper and ours. The main new elements, in comparison to Chen and Zhang (2016), are the proof of crucial Theorem 3.2, the proof of Lemma 4.4, the estimates (4.9-4.11) and the proof of lower bound estimates of $p^{A}(t, x, y)$. It is worth pointing out that in our paper we have shown that the transition density $p^{A}(t, x, y)$ satisfies the equation (1.4) for all $x, y \in \mathbb{R}^{d}$ while in Chen and Zhang (2016) it is shown that the heat kernel $p_{\alpha}^{\kappa}(t, x, y)$ satisfies the analogous equation only when $x \neq y$. A similar remark concerns gradient estimates of $p^{A}(t, x, y)$, which we managed to show for all $x, y \in \mathbb{R}^{d}$. On the other hand, we were able to prove gradient estimates of $p^{A}(t, x, y)$ only for $\alpha \in(1,2)$, while in the latter paper gradient estimates were obtained for $\alpha \in[1,2)$. It is worth mentioning that quite recently a very interesting generalization of the results from Chen and Zhang (2016) appeared in Kim et al. (2018).

The problem of estimates of transition densities for jump Lévy and Lévy-type processes has been intensively studied in recent years see e.g. Chen and Zhang (2016); Kim et al. (2018); Bogdan et al. (2014); Chen and Kumagai (2008); Chen et al. (2011); Knopova and Schilling (2012); Kaleta and Sztonyk (2015); Knopova (2013); Mimica (2012); Grzywny and Szczypkowski (2017, 2018); Knopova and Kulik $(2017,2011)$. However, relatively few results concern processes with jump kernels which are not comparable to isotropic ones. We have already mentioned here 
the paper Bogdan et al. (2017). One should also mention the papers by Bogdan, Kaleta and Sztonyk (see Bogdan and Sztonyk, 2007; Kaleta and Sztonyk, 2017, 2015; Sztonyk, 2017) but they only concern heat kernels of translation invariant generators and convolution semigroups for which the existence and many properties follow by Fourier methods. There are also known estimates of anisotropic non-convolution heat kernels given in Sztonyk (2010a); Kaleta and Sztonyk (2013) however these are obtained under the assumption that the jump kernel is dominated by that of the rotation invariant stable process. For estimates of derivatives of Lévy densities we refer the reader to Sztonyk (2010b); Bogdan and Jakubowski (2007); Schilling et al. (2012); Kaleta and Sztonyk (2015); Kulczycki and Ryznar (2016); Knopova (2013); Chen and Zhang (2016); Knopova and Kulik (2018); Kulik (2018); Grzywny and Szczypkowski $(2017,2018)$. Very important results concerning existence and Feller property of solutions to Lévy driven SDEs were obtained by F. Kühn (see e.g. Kühn (2012); Kühn (2017, 2018a,b)). Interesting results about weak Euler approximation for solutions of some Lévy driven SDEs were obtained in Mikulevičius and Zhang (2011, 2015).

Some estimates of transition densities for processes which are solutions of systems of SDEs driven by Lévy processes with singular Lévy measures were obtained in Picard (1997, 1995/97, 1996); Ishikawa (2001). However, the results from Picard (1997), when applied to system (1.1), do not imply such sharp estimates which are obtained in Theorem 1.1. In particular, they can be applied to system (1.1) only when $x \rightarrow a_{i i}(x)$ are $C^{\infty}\left(\mathbb{R}^{d}\right)$ functions. What is more, even in this case, the upper bound estimates are of the form $\sup _{x, y \in \mathbb{R}^{d}} p^{A}(t, x, y) \leq c t^{-d / \alpha}$, while the lower bound estimates of $p^{A}(t, x, y)$ are also much less precise than ours. They are precise only for $x=y$, in which case it follows from Picard (1997) that $p^{A}(t, x, x) \approx t^{-d / \alpha}$. The results from Picard $(1995 / 97,1996)$; Ishikawa (2001) cannot be applied to system (1.1).

The paper is organized as follows. In Section 2 we introduce the notation and collect known facts needed in the sequel. In Section 3 we construct the function $p^{A}(t, x, y)$ in terms of the perturbation series $q(t, x, y)=\sum_{n=0}^{\infty} q_{n}(t, x, y)$ using Picard's iteration. In Theorem 3.2 we obtain the estimates of $q(t, x, y)$ which are absolutely crucial for the rest of the paper. In Section 4 we show that the semigroup defined by $P_{t}^{A} f(x)=\int_{\mathbb{R}^{d}} p^{A}(t, x, y) f(y) d y$ is a Feller semigroup. Next, applying the results from Bass and Chen (2006), we argue that $p^{A}(t, x, y)$ is, in fact, the transition density of the solution of system (1.1) and we prove most parts of the main theorem. In Section 5 we show lower bound estimates of $p^{A}(t, x, y)$ by using probabilistic arguments.

\section{Preliminaries}

All constants appearing in this paper are positive and finite. In the whole paper we fix $T>0, d \geq 2, d \in \mathbb{N}, \alpha \in(0,2), b_{1}, b_{2}, b_{3}, \beta$, where $b_{1}, b_{2}, b_{3}, \beta$ appear in (1.2) and (1.3). We adopt the convention that constants denoted by $c$ (or $c_{1}, c_{2}, \ldots$ ) may change their value from one use to the next. In the whole paper, unless is explicitly stated otherwise, we understand that constants denoted by $c$ (or $c_{1}, c_{2}, \ldots$ ) depend on $T, d, \alpha, b_{1}, b_{2}, b_{3}, \beta$. We also understand that they may depend on the choice of the constant $\gamma \in(0, \beta)$ (or $\gamma \in(0, \alpha \wedge 1)$ ). We write $f(x) \approx g(x)$ for $x \in A$ if 
$f, g \geq 0$ on $A$ and there is a constant $c \geq 1$ such that $c^{-1} f(x) \leq g(x) \leq c f(x)$ for $x \in A$.

Denote

$$
\sigma_{i}(x)=a_{i i}^{\alpha}(x) .
$$

Note that there exists $c$ such that for any $x, y \in \mathbb{R}^{d}$ we have

$$
\left|\sigma_{i}(x)-\sigma_{i}(y)\right| \leq c\left(|x-y|^{\beta} \wedge 1\right) .
$$

By simple change of variable we get

$$
\mathcal{L} f(x)=\sum_{i=1}^{d} \lim _{\varepsilon \rightarrow 0^{+}} \frac{\mathcal{A}_{\alpha}}{2} \int_{\left|z_{i}\right|>\varepsilon}\left[f\left(x+e_{i} z_{i}\right)+f\left(x-e_{i} z_{i}\right)-2 f(x)\right] \sigma_{i}(x) \frac{d z_{i}}{\left|z_{i}\right|^{1+\alpha}} .
$$

Let us introduce some notation used in Chen and Zhang (2016). For a function $f: \mathbb{R}^{d} \rightarrow \mathbb{R}$ we denote

$$
\delta_{f}(x, z)=f(x+z)+f(x-z)-2 f(x) .
$$

Similarly, for a function $f: \mathbb{R}_{+} \times \mathbb{R}^{d} \rightarrow \mathbb{R}$ we write

$$
\delta_{f}(t, x, z)=f(t, x+z)+f(t, x-z)-2 f(t, x) .
$$

We also denote

$$
\rho_{\gamma}^{\beta}(t, x)=t^{\gamma / \alpha}\left(|x|^{\beta} \wedge 1\right)\left(t^{1 / \alpha}+|x|\right)^{-1-\alpha}, \quad t>0, x \in \mathbb{R} .
$$

It is well known that

$$
g_{t}(x) \approx \rho_{\alpha}^{0}(t, x), \quad t>0, x \in \mathbb{R} .
$$

One of the most important tools used in our paper are convolution estimates Chen and Zhang (2016, (2.3-2.4)). They are similar to Kolokoltsov (2000, Lemma 1.4) and Xie and Zhang (2014, Lemma 2.3). In Chen and Zhang (2016) they are stated for $t \in(0,1]$. It is easy to check that they hold also for $t \in(0, T]$. For reader's convenience we collected them in Lemma 2.1.

Lemma 2.1. (i) There is $C=C(\alpha)$ such that for any $t>0$ and any $\beta_{1} \in[0, \alpha / 2], \gamma_{1} \in \mathbb{R}$,

$$
\int_{\mathbb{R}} \rho_{\gamma_{1}}^{\beta_{1}}(t, z) d z \leq C t^{\frac{\gamma_{1}+\beta_{1}-\alpha}{\alpha}} .
$$

(ii) For $T>0$ there is $C=C(\alpha, T)$ such that for any $0<s<t \leq T, x \in \mathbb{R}$ and any $\beta_{1}, \beta_{2} \in[0, \alpha / 4], \gamma_{1}, \gamma_{2} \in \mathbb{R}$ we have

$$
\begin{aligned}
& \int_{\mathbb{R}} \rho_{\gamma_{1}}^{\beta_{1}}(t-s, x-z) \rho_{\gamma_{2}}^{\beta_{2}}(s, z) d z \\
& \leq C\left[(t-s)^{\frac{\gamma_{1}+\beta_{1}+\beta_{2}-\alpha}{\alpha}} s^{\frac{\gamma_{2}}{\alpha}}+(t-s)^{\frac{\gamma_{1}}{\alpha}} s^{\frac{\gamma_{2}+\beta_{1}+\beta_{2}-\alpha}{\alpha}}\right] \rho_{0}^{0}(t, x) \\
& +C\left[(t-s)^{\frac{\gamma_{1}+\beta_{1}-\alpha}{\alpha}} s^{\frac{\gamma_{2}}{\alpha}} \rho_{0}^{\beta_{2}}(t, x)+(t-s)^{\frac{\gamma_{1}}{\alpha}} s^{\frac{\gamma_{2}+\beta_{2}-\alpha}{\alpha}} \rho_{0}^{\beta_{1}}(t, x)\right] .
\end{aligned}
$$

(iii) For $T>0$ there is $C=C(\alpha, T)$ such that for any $0<t \leq T, x \in \mathbb{R}$ and any $\beta_{1}, \beta_{2} \in[0, \alpha / 4], \gamma_{1}, \gamma_{2} \in \mathbb{R}$ with $\gamma_{1}+\beta_{1}>0$ and $\gamma_{2}+\beta_{2}>0$ we have

$$
\begin{aligned}
& \int_{0}^{t} \int_{\mathbb{R}} \rho_{\gamma_{1}}^{\beta_{1}}(t-s, x-z) \rho_{\gamma_{2}}^{\beta_{2}}(s, z) d z d s \\
& \leq C \mathcal{B}\left(\frac{\gamma_{1}+\beta_{1}}{\alpha}, \frac{\gamma_{2}+\beta_{2}}{\alpha}\right)\left(\rho_{\gamma_{1}+\gamma_{2}+\beta_{1}+\beta_{2}}^{0}+\rho_{\gamma_{1}+\gamma_{2}+\beta_{2}}^{\beta_{1}}+\rho_{\gamma_{1}+\gamma_{2}+\beta_{1}}^{\beta_{2}}\right)(t, x),
\end{aligned}
$$


where $\mathcal{B}(u, w)$ is the Beta function.

Similarly as in Chen and Zhang (2016) we introduce, for $y \in \mathbb{R}^{d}$, the freezing operator $\mathcal{L}^{y}$ by

$$
\mathcal{L}^{y} f(x)=\frac{\mathcal{A}_{\alpha}}{2} \sum_{i=1}^{d} \int_{\mathbb{R}} \delta_{f}\left(x, e_{i} z_{i}\right) \sigma_{i}(y) \frac{d z_{i}}{\left|z_{i}\right|^{1+\alpha}}
$$

and

$$
\mathcal{L}^{y} f(t, x)=\frac{\mathcal{A}_{\alpha}}{2} \sum_{i=1}^{d} \int_{\mathbb{R}} \delta_{f}\left(t, x, e_{i} z_{i}\right) \sigma_{i}(y) \frac{d z_{i}}{\left|z_{i}\right|^{1+\alpha}}
$$

Put

$$
p_{y}(t, x)=\prod_{i=1}^{d} \frac{1}{a_{i i}(y)} g_{t}\left(\frac{x_{i}}{a_{i i}(y)}\right) .
$$

It is clear that $p_{y}(t, x)$ is the heat kernel of the operator $\mathcal{L}^{y}$. In particular, we have

$$
\frac{\partial}{\partial t} p_{y}(t, x)=\mathcal{L}^{y} p_{y}(t, x), \quad t>0, x, y \in \mathbb{R}^{d}
$$

In the sequel we will use the following standard estimate. For any $\gamma \in(0,1]$ there exists $c=c(\gamma)$ such that for any $\theta \geq 1$ we have

$$
\int_{0}^{t}(t-s)^{\gamma-1} s^{\theta-1} d s \leq \frac{c}{\theta^{\gamma}} t^{(\gamma-1)+(\theta-1)+1} .
$$

We use the notation $\mathbb{N}_{0}=\mathbb{N} \cup\{0\}$.

\section{Upper bound estimates}

The main aim of this section is to construct the function $p^{A}(t, x, y)$. This is done by using Levi's method. We recall that this method was applied by Kochuber (1988) in the framework of pseudodifferential operators. In the recent years it was used in several papers to study gradient and Schrödinger perturbations of fractional Laplacians see e.g. Bogdan and Jakubowski (2007); Jakubowski and Szczypkowski (2012, 2010); Chen and Hu (2015); Chen et al. (2012, 2015); Xie and Zhang (2014). As we have already mentioned we use the approach from Chen and Zhang (2016). It is worth adding that in there, in contrast to previous papers, a new way of "freezing" coefficient was used.

Now, we briefly present the main steps used in this section. We define $p^{A}(t, x, y)$ by (3.2). Heuristically, $p^{A}(t, x, y)$ is equal to the transition density $p_{y}(t, x-y)$ (of the process with the "frozen" jump measure corresponding to the generator $\mathcal{L}^{y}$ ) plus some correction $\int_{0}^{t} \int_{\mathbb{R}^{d}} p_{z}(t-s, x, z) q(s, z, y) d z d s$, which is given in terms of the perturbation series $q(t, x, y)=\sum_{n=0}^{\infty} q_{n}(t, x, y)$. The most difficult result in this section is Theorem 3.2 which gives upper bound estimates of $q(t, x, y)$. Due to a different structure of the generator $\mathcal{L}$ in comparison to the Lévy-type operator $\mathcal{L}_{\alpha}^{\kappa}$ from Chen and Zhang (2016) there are essential differences between our proof and analogous proof in Chen and Zhang (2016), see in particular the definition of the auxiliary function $H_{k}^{L}(t, x, y)$ and the induction proof of (3.6). The next important step in this section is Theorem 3.9, where we derive Hölder type estimates of $q(t, x, y)$. We also show crucial Lemma 3.14, which is the main step in obtaining gradient estimates of $p^{A}(t, x, y)$. 
For $x, y \in \mathbb{R}^{d}, t>0$, let

$$
q_{0}(t, x, y)=\left(\mathcal{L}^{x}-\mathcal{L}^{y}\right) p_{y}(t, \cdot)(x-y)
$$

and for $n \in \mathbb{N}$ let

$$
q_{n}(t, x, y)=\int_{0}^{t} \int_{\mathbb{R}^{d}} q_{0}(t-s, x, z) q_{n-1}(s, z, y) d z d s .
$$

For $x, y \in \mathbb{R}^{d}, t>0$ we define

$$
q(t, x, y)=\sum_{n=0}^{\infty} q_{n}(t, x, y)
$$

and

$$
p^{A}(t, x, y)=p_{y}(t, x-y)+\int_{0}^{t} \int_{\mathbb{R}^{d}} p_{z}(t-s, x, z) q(s, z, y) d z d s .
$$

By Chen and Zhang (2016, (2.28)) and (2.2) one easily obtains

Lemma 3.1. For any $t \in(0, T]$ and $x, y \in \mathbb{R}^{d}$ we have

$$
\sum_{k=1}^{d} \int_{\mathbb{R}}\left|\delta_{p_{y}}\left(t, x, z_{k} e_{k}\right)\right| \frac{d z_{k}}{\left|z_{k}\right|^{1+\alpha}} \leq c t^{d-1} \prod_{i=1}^{d} \rho_{0}^{0}\left(t, x_{i}\right) .
$$

An immediate consequence of the above lemma and (2.6) is the following estimate

$$
\left|\frac{\partial}{\partial t} p_{y}(t, x)\right| \leq c t^{d-1} \prod_{i=1}^{d} \rho_{0}^{0}\left(t, x_{i}\right)
$$

for $t \in(0, T], x, y \in \mathbb{R}^{d}$.

Theorem 3.2. The series $\sum_{n=0}^{\infty} q_{n}(t, x, y)$ is absolutely and locally uniformly convergent on $(0, T] \times \mathbb{R}^{d} \times \mathbb{R}^{d}$. For any $x, y \in \mathbb{R}^{d}, t \in(0, T]$ we have

$$
|q(t, x, y)| \leq c t^{d-1}\left[\prod_{i=1}^{d} \rho_{0}^{0}\left(t, x_{i}-y_{i}\right)\right]\left[t^{\beta / \alpha}+\sum_{m=1}^{d}\left(\left|x_{m}-y_{m}\right|^{\beta} \wedge 1\right)\right] .
$$

Moreover, $q(t, x, y)$ is jointly continuous in $(t, x, y) \in(0, T] \times \mathbb{R}^{d} \times \mathbb{R}^{d}$.

Proof: By (2.1) and then Lemma 3.1 we get

$$
\begin{aligned}
\left|q_{0}(t, x, y)\right| & \leq \frac{\mathcal{A}_{\alpha}}{2} \sum_{k=1}^{d} \int_{\mathbb{R}}\left|\delta_{p_{y}}\left(t, x-y, e_{k} z_{k}\right)\right|\left|\sigma_{k}(x)-\sigma_{k}(y)\right| \frac{d z_{k}}{\left|z_{k}\right|^{1+\alpha}} \\
& \leq c\left(|x-y|^{\beta} \wedge 1\right) \sum_{k=1}^{d} \int_{\mathbb{R}}\left|\delta_{p_{y}}\left(t, x-y, e_{k} z_{k}\right)\right| \frac{d z_{k}}{\left|z_{k}\right|^{1+\alpha}} \\
& \leq M t^{d-1}\left[\sum_{m=1}^{d}\left(\left|x_{m}-y_{m}\right|^{\beta} \wedge 1\right)\right]\left[\prod_{k=1}^{d} \rho_{0}^{0}\left(t, x_{k}-y_{k}\right)\right],
\end{aligned}
$$

where $M=M\left(T, d, \alpha, b_{1}, b_{2}, b_{3}, \beta\right)$.

Put

$$
\mathrm{I}=\left\{L=\left(l_{1}, \ldots, l_{d}\right): \forall i \in\{1, \ldots, d\} l_{i}=0 \text { or } l_{i}=\beta\right\}
$$


For any $L=\left(l_{1}, \ldots, l_{d}\right) \in \mathrm{I}$ denote

$$
|L|=\frac{1}{\beta} \sum_{i=1}^{d} l_{i}
$$

For $k \in \mathbb{N}_{0}$ and $L=\left(l_{1}, \ldots, l_{d}\right) \in \mathrm{I}$ put

$$
H_{k}^{L}(t, x, y)=t^{d-1+k \beta / \alpha}\left[\prod_{i=1}^{d} \rho_{0}^{0}\left(t, x_{i}-y_{i}\right)\right]\left[\prod_{j=1}^{d}\left(\left|x_{j}-y_{j}\right|^{l_{j}} \wedge 1\right)\right] .
$$

We will show that there is $C=C\left(T, d, \alpha, b_{1}, b_{2}, b_{3}, \beta\right)$ such that for any $n \in \mathbb{N}_{0}$, $x, y \in \mathbb{R}^{d}, t \in(0, T]$,

$$
\left|q_{n}(t, x, y)\right| \leq \frac{M C^{n}}{((n+1) !)^{\beta / \alpha}} \sum_{\substack{k \in \mathbb{N}_{0}, L \in \mathrm{I} \\ k+|L|=n+1}} H_{k}^{L}(t, x, y),
$$

where $M$ is the constant from (3.5). Let

$$
D(t, x, y, m, k, L)=M \int_{0}^{t} \int_{\mathbb{R}^{d}} H_{0}^{L_{m}}(t-s, x, z) H_{k}^{L}(s, z, y) d z d s,
$$

where $L_{m} \in \mathrm{I}$ is such that $l_{m}=\beta$ and $\left|L_{m}\right|=1$. Observe that (3.5) can be rewritten as

$$
\left|q_{0}(t, x, y)\right| \leq M \sum_{m=1}^{d} H_{0}^{L_{m}}(t, x, y) .
$$

We will prove (3.6) by induction. The main step consists of proving that for any $n \in \mathbb{N}$,

$$
\sum_{m=1}^{d} \sum_{\substack{k \in \mathbb{N}_{0}, L \in \mathrm{I} \\ k+|L|=n+1}} D(t, x, y, m, k, L) \leq \frac{C}{(n+2)^{\beta / \alpha}} \sum_{\substack{k \in \mathbb{N}_{0}, L \in \mathrm{I} \\ k+|L|=n+2}} H_{k}^{L}(t, x, y) .
$$

For $n=0$ the estimate (3.6) holds by (3.7).

Assume that (3.6) holds for some $n \in \mathbb{N}_{0}$. By (3.1), (3.7) and our induction hypothesis we obtain

$$
\left|q_{n+1}(t, x, y)\right| \leq \frac{M C^{n}}{((n+1) !)^{\beta / \alpha}} \sum_{m=1}^{d} \sum_{\substack{k \in \mathbb{N}_{0}, L \in \mathrm{I} \\ k+|L|=n+1}} D(t, x, y, m, k, L) .
$$

Then, if (3.8) is true, then (3.6) holds for $n+1$. Hence in order to complete the proof it is enough to show (3.8).

To this end we consider 3 cases.

Case 1. $L=(0, \ldots, 0), k=n+1$.

We have

$$
D(t, x, y, m, k, L)=M \int_{0}^{t} s^{n \beta / \alpha}\left[\prod_{\substack{i=1 \\ i \neq m}}^{d} \int_{\mathbb{R}} \rho_{\alpha}^{0}\left(t-s, x_{i}-z_{i}\right) \rho_{\alpha}^{0}\left(s, z_{i}-y_{i}\right) d z_{i}\right]
$$




$$
\times \int_{\mathbb{R}} \rho_{0}^{\beta}\left(t-s, x_{m}-z_{m}\right) \rho_{\beta}^{0}\left(s, z_{m}-y_{m}\right) d z_{m} d s .
$$

By (2.4), we obtain

$$
\int_{\mathbb{R}} \rho_{\alpha}^{0}\left(t-s, x_{i}-z_{i}\right) \rho_{\alpha}^{0}\left(s, z_{i}-y_{i}\right) d z_{i} \leq c \rho_{\alpha}^{0}\left(t, x_{i}-y_{i}\right)
$$

and

$$
\begin{aligned}
& \int_{\mathbb{R}} \rho_{0}^{\beta}\left(t-s, x_{m}-z_{m}\right) \rho_{\beta}^{0}\left(s, z_{m}-y_{m}\right) d z_{m} \\
& \leq c\left[(t-s)^{(\beta-\alpha) / \alpha} s^{\beta / \alpha} \rho_{0}^{0}\left(t, x_{m}-y_{m}\right)+s^{(2 \beta-\alpha) / \alpha} \rho_{0}^{0}\left(t, x_{m}-y_{m}\right)\right. \\
& \left.\quad+s^{(\beta-\alpha) / \alpha} \rho_{0}^{\beta}\left(t, x_{m}-y_{m}\right)\right] .
\end{aligned}
$$

Hence

$$
\begin{aligned}
D(t, x, y, m, k, L) \leq & c t^{d-1}\left[\prod_{i=1}^{d} \rho_{0}^{0}\left(t, x_{i}-y_{i}\right)\right] \\
\times & {\left[\int_{0}^{t}(t-s)^{(\beta-\alpha) / \alpha} s^{(n+1) \beta / \alpha} d s+\int_{0}^{t} s^{((n+2) \beta-\alpha) / \alpha} d s\right.} \\
& \left.+\int_{0}^{t} s^{((n+1) \beta-\alpha) / \alpha} d s\left(\left|x_{m}-y_{m}\right|^{\beta} \wedge 1\right)\right] .
\end{aligned}
$$

By (2.7) this implies that

$$
\begin{aligned}
D(t, x, y, m, k, L) \leq & \frac{c}{(n+1)^{\beta / \alpha}} t^{d-1}\left[\prod_{i=1}^{d} \rho_{0}^{0}\left(t, x_{i}-y_{i}\right)\right] \\
& \times\left[t^{(n+2) \beta / \alpha}+t^{(n+1) \beta / \alpha}\left(\left|x_{m}-y_{m}\right|^{\beta} \wedge 1\right)\right] .
\end{aligned}
$$

Case 2. $L=\left(l_{1}, \ldots, l_{d}\right) \neq(0, \ldots, 0), l_{m}=0$.

Put $Z(L)=\left\{i \in\{1, \ldots, d\}: l_{i}=\beta\right\}$ and $i(L)=\inf Z(L)$. Clearly $m \notin Z(L)$. We have

$$
\begin{aligned}
& D(t, x, y, m, k, L)=M \int_{0}^{t} \int_{\mathbb{R}} \rho_{0}^{\beta}\left(t-s, x_{m}-z_{m}\right) \rho_{\alpha}^{0}\left(s, z_{m}-y_{m}\right) d z_{m} \\
& \times \int_{\mathbb{R}} \rho_{\alpha}^{0}\left(t-s, x_{i(L)}-z_{i(L)}\right) \rho_{0}^{\beta}\left(s, z_{i(L)}-y_{i(L)}\right) d z_{i(L)} \\
& \times\left[\prod_{\substack{i \in Z(L) \\
i \neq i(L)}} \int_{\mathbb{R}} \rho_{\alpha}^{0}\left(t-s, x_{i}-z_{i}\right) \rho_{\alpha}^{\beta}\left(s, z_{i}-y_{i}\right) d z_{i}\right] \\
& \times\left[\prod_{\substack{i \notin Z(L) \\
i \neq m}} \int_{\mathbb{R}} \rho_{\alpha}^{0}\left(t-s, x_{i}-z_{i}\right) \rho_{\alpha}^{0}\left(s, z_{i}-y_{i}\right) d z_{i}\right] s^{k \beta / \alpha} d s .
\end{aligned}
$$

By (2.4) this is bounded from above by

$$
c \int_{0}^{t}\left[(t-s)^{(\beta-\alpha) / \alpha} s \rho_{0}^{0}\left(t, x_{m}-y_{m}\right)+t^{\beta / \alpha} \rho_{0}^{0}\left(t, x_{m}-y_{m}\right)+\rho_{0}^{\beta}\left(t, x_{m}-y_{m}\right)\right]
$$




$$
\begin{aligned}
& \times\left[t^{\beta / \alpha} \rho_{0}^{0}\left(t, x_{i(L)}-y_{i(L)}\right)+t s^{(\beta-\alpha) / \alpha} \rho_{0}^{0}\left(t, x_{i(L)}-y_{i(L)}\right)+\rho_{0}^{\beta}\left(t, x_{i(L)}-y_{i(L)}\right)\right] \\
& \times\left[\prod_{\substack{i \in Z(L) \\
i \neq i(L)}}\left[t^{(\alpha+\beta) / \alpha} \rho_{0}^{0}\left(t, x_{i}-y_{i}\right)+t \rho_{0}^{\beta}\left(t, x_{i}-y_{i}\right)\right]\right] \\
& \times\left[\prod_{\substack{i \notin Z(L) \\
i \neq m}} t \rho_{0}^{0}\left(t, x_{i}-y_{i}\right)\right] s^{k \beta / \alpha} d s .
\end{aligned}
$$

Note that $\# Z(L)=|L|$. We have

$$
\begin{aligned}
& \prod_{\substack{i \in Z(L) \\
i \neq i(L)}}\left[t^{(\alpha+\beta) / \alpha} \rho_{0}^{0}\left(t, x_{i}-y_{i}\right)+t \rho_{0}^{\beta}\left(t, x_{i}-y_{i}\right)\right] \leq\left. c\right|^{|L|-1}\left(\prod_{\substack{i \in Z(L) \\
i \neq i(L)}} \rho_{0}^{0}\left(t, x_{i}-y_{i}\right)\right) \\
& \times \sum_{\substack{r \leq|L|-1 \\
r \in \mathbb{N}_{0}}} \sum_{\left\{k_{1}, \ldots, k_{r}\right\} \subset Z(L) \backslash\{i(L)\}} t^{(|L|-r-1) \beta / \alpha} \prod_{i=1}^{r}\left(\left|x_{k_{i}}-y_{k_{i}}\right|^{\beta} \wedge 1\right),
\end{aligned}
$$

where for $r=0$ we understand that $\prod_{i=1}^{r}\left(\left|x_{k_{i}}-y_{k_{i}}\right|^{\beta} \wedge 1\right)=1$. It follows that

$$
\begin{aligned}
& D(t, x, y, m, k, L) \leq c t^{d-2}\left[\prod_{i=1}^{d} \rho_{0}^{0}\left(t, x_{i}-y_{i}\right)\right] \\
& \times \sum_{\substack{r \leq|L|-1 \\
r \in \mathbb{N}_{0}}} \sum_{\left\{k_{1}, \ldots, k_{r}\right\} \subset Z(L) \backslash\{i(L)\}} t^{(|L|-r-1) \beta / \alpha}\left[\prod_{i=1}^{r}\left(\left|x_{k_{i}}-y_{k_{i}}\right|^{\beta} \wedge 1\right)\right] \\
& \times\left[t^{(\alpha+\beta) / \alpha} \int_{0}^{t}(t-s)^{(\beta-\alpha) / \alpha} s^{k \beta / \alpha} d s+t \int_{0}^{t}(t-s)^{(\beta-\alpha) / \alpha} s^{(\beta+k \beta) / \alpha} d s\right. \\
& +t \int_{0}^{t}(t-s)^{(\beta-\alpha) / \alpha} s^{k \beta / \alpha} d s\left(\left|x_{i(L)}-y_{i(L)}\right|^{\beta} \wedge 1\right) \\
& +t^{2 \beta / \alpha} \int_{0}^{t} s^{k \beta / \alpha} d s+t^{(\alpha+\beta) / \alpha} \int_{0}^{t} s^{(\beta-\alpha+k \beta) / \alpha} d s \\
& +t^{\beta / \alpha} \int_{0}^{t} s^{k \beta / \alpha} d s\left(\left|x_{i(L)}-y_{i(L)}\right|^{\beta} \wedge 1\right) \\
& \times\left[t^{\beta / \alpha} \int_{0}^{t} s^{k \beta / \alpha} d s+t \int_{0}^{t} s^{(\beta-\alpha+k \beta) / \alpha} d s+\int_{0}^{t} s^{k \beta / \alpha} d s\left(\left|x_{i(L)}-y_{i(L)}\right|^{\beta} \wedge 1\right)\right] \\
& \left.\times\left(\left|x_{m}-y_{m}\right|^{\beta} \wedge 1\right)\right] .
\end{aligned}
$$

Using this and (2.7) we get

$D(t, x, y, m, k, L) \leq c t^{d-2}\left[\prod_{i=1}^{d} \rho_{0}^{0}\left(t, x_{i}-y_{i}\right)\right]$ 


$$
\begin{aligned}
& \times \sum_{\substack{r \leq|L|-1 \\
r \in \mathbb{N}_{0}}} \sum_{\left\{k_{1}, \ldots, k_{r}\right\} \subset Z(L) \backslash\{i(L)\}} t^{(|L|-r-1) \beta / \alpha}\left[\prod_{i=1}^{r}\left(\left|x_{k_{i}}-y_{k_{i}}\right|^{\beta} \wedge 1\right)\right] \\
& \times \frac{1}{(k+1)^{\beta / \alpha}}\left[t^{(\alpha+k \beta+2 \beta) / \alpha}+t^{(\alpha+k \beta+\beta) / \alpha}\left(\left|x_{i(L)}-y_{i(L)}\right|^{\beta} \wedge 1\right)\right. \\
& \left.t^{(\alpha+k \beta+\beta) / \alpha}\left(\left|x_{m}-y_{m}\right|^{\beta} \wedge 1\right)+t^{(\alpha+k \beta) / \alpha}\left(\left|x_{i(L)}-y_{i(L)}\right|^{\beta} \wedge 1\right)\left(\left|x_{m}-y_{m}\right|^{\beta} \wedge 1\right)\right] .
\end{aligned}
$$

Note that $k+|L|=n+1$. It follows that

$$
\begin{aligned}
& D(t, x, y, m, k, L) \leq \frac{c t^{d-1}}{(k+1)^{\beta / \alpha}}\left[\prod_{i=1}^{d} \rho_{0}^{0}\left(t, x_{i}-y_{i}\right)\right] \\
& \times \sum_{\substack{r \leq|L|+1 \\
r \in \mathbb{N}_{0}}} \sum_{\left\{k_{1}, \ldots, k_{r}\right\} \subset Z(L) \cup\{m\}} t^{(n+2-r) \beta / \alpha} \prod_{i=1}^{r}\left(\left|x_{k_{i}}-y_{k_{i}}\right|^{\beta} \wedge 1\right) .
\end{aligned}
$$

Case 3. $L=\left(l_{1}, \ldots, l_{d}\right), l_{m}=\beta$.

We have

$$
\begin{aligned}
& D(t, x, y, m, k, L)=M \int_{0}^{t} \int_{\mathbb{R}} \rho_{0}^{\beta}\left(t-s, x_{m}-z_{m}\right) \rho_{0}^{\beta}\left(s, z_{m}-y_{m}\right) d z_{m} \\
& \times\left[\prod_{\substack{i \in Z(L) \\
i \neq m}} \int_{\mathbb{R}} \rho_{\alpha}^{0}\left(t-s, x_{i}-z_{i}\right) \rho_{\alpha}^{\beta}\left(s, z_{i}-y_{i}\right) d z_{i}\right] \\
& \times\left[\prod_{i \notin Z(L)} \int_{\mathbb{R}} \rho_{\alpha}^{0}\left(t-s, x_{i}-z_{i}\right) \rho_{\alpha}^{0}\left(s, z_{i}-y_{i}\right) d z_{i}\right] s^{k \beta / \alpha} d s .
\end{aligned}
$$

By (2.4), this is bounded from above by

$$
\begin{aligned}
& c \int_{0}^{t}\left[(t-s)^{(2 \beta-\alpha) / \alpha} \rho_{0}^{0}\left(t, x_{m}-y_{m}\right)+s^{(2 \beta-\alpha) / \alpha} \rho_{0}^{0}\left(t, x_{m}-y_{m}\right)\right. \\
& \left.+(t-s)^{(\beta-\alpha) / \alpha} \rho_{0}^{\beta}\left(t, x_{m}-y_{m}\right)+s^{(\beta-\alpha) / \alpha} \rho_{0}^{\beta}\left(t, x_{m}-y_{m}\right)\right] \\
& \times\left[\prod_{\substack{i \in Z(L) \\
i \neq m}}\left[t^{(\alpha+\beta) / \alpha} \rho_{0}^{0}\left(t, x_{i}-y_{i}\right)+t \rho_{0}^{\beta}\left(t, x_{i}-y_{i}\right)\right]\right] \\
& \times\left[\prod_{i \notin Z(L)} t \rho_{0}^{0}\left(t, x_{i}-y_{i}\right)\right] s^{k \beta / \alpha} d s .
\end{aligned}
$$

Using similar reasoning as in (3.11) this is bounded from above by

$$
c t^{d-1}\left[\prod_{i=1}^{d} \rho_{0}^{0}\left(t, x_{i}-y_{i}\right)\right]
$$




$$
\begin{aligned}
& \times \sum_{r \leq|L|-1} \sum_{\substack{r \in \mathbb{N}_{0} \\
\left\{k_{1}, \ldots, k_{r}\right\} \subset Z(L) \backslash\{m\}}} t^{(|L|-r-1) \beta / \alpha} \prod_{i=1}^{r}\left(\left|x_{k_{i}}-y_{k_{i}}\right|^{\beta} \wedge 1\right) \\
& {\left[\int_{0}^{t}(t-s)^{(2 \beta-\alpha) / \alpha} s^{k \beta / \alpha} d s+\int_{0}^{t} s^{(2 \beta-\alpha+k \beta) / \alpha} d s\right.} \\
& +\int_{0}^{t}(t-s)^{(\beta-\alpha) / \alpha} s^{k \beta / \alpha} d s\left(\left|x_{m}-y_{m}\right|^{\beta} \wedge 1\right) \\
& \left.\int_{0}^{t} s^{(\beta-\alpha+k \beta) / \alpha} d s\left(\left|x_{m}-y_{m}\right|^{\beta} \wedge 1\right)\right]
\end{aligned}
$$

By (2.7) it follows that

$$
\begin{aligned}
& D(t, x, y, m, k, L) \leq c t^{d-1}\left[\prod_{i=1}^{d} \rho_{0}^{0}\left(t, x_{i}-y_{i}\right)\right] \\
& \times \sum_{\substack{r \leq|L|-1 \\
r \in \mathbb{N}_{0}}} \sum_{\left\{k_{1}, \ldots, k_{r}\right\} \subset Z(L) \backslash\{m\}} t^{(|L|-r-1) \beta / \alpha} \prod_{i=1}^{r}\left(\left|x_{k_{i}}-y_{k_{i}}\right|^{\beta} \wedge 1\right) \\
& \frac{1}{(k+1)^{\beta / \alpha}}\left[t^{(k \beta+2 \beta) / \alpha}+t^{(k \beta+\beta) / \alpha}\left(\left|x_{m}-y_{m}\right|^{\beta} \wedge 1\right)\right]
\end{aligned}
$$

Hence

$$
\begin{aligned}
& D(t, x, y, m, k, L) \leq \frac{c t^{d-1}}{(k+1)^{\beta / \alpha}}\left[\prod_{i=1}^{d} \rho_{0}^{0}\left(t, x_{i}-y_{i}\right)\right] \\
& \times \sum_{\substack{r \leq|L| \\
r \in \mathbb{N}_{0}}} \sum_{\left\{k_{1}, \ldots, k_{r}\right\} \subset Z(L)} t^{(n+2-r) \beta} \prod_{i=1}^{r}\left(\left|x_{k_{i}}-y_{k_{i}}\right|^{\beta} \wedge 1\right) .
\end{aligned}
$$

Recall that $n+1=k+|L|,|L| \leq d$, so $k \geq n+1-d$. Hence $\frac{1}{(k+1)^{\beta / \alpha}} \leq \frac{c}{(n+2)^{\beta / \alpha}}$, where $c=c(d)$. Consequently, (3.9), (3.10), (3.12), (3.13) gives that (3.8) holds, which finishes the induction proof.

From (3.6) we immediately obtain that for any $n \in \mathbb{N}_{0}$

$$
\left|q_{n}(t, x, y)\right| \leq \frac{c C^{n}}{((n+1) !)^{\beta / \alpha}} t^{d-1}\left[\prod_{i=1}^{d} \rho_{0}^{0}\left(t, x_{i}-y_{i}\right)\right]\left[t^{\beta / \alpha}+\sum_{m=1}^{d}\left(\left|x_{m}-y_{m}\right|^{\beta} \wedge 1\right)\right] .
$$

It follows that $\sum_{n=0}^{\infty} q_{n}(t, x, y)$ is absolutely and locally uniformly convergent on $(0, T] \times \mathbb{R}^{d} \times \mathbb{R}^{d}$ and (3.4) holds.

By the properties of $p_{y}(t, x)$ it is easy to justify that $q_{0}(t, x, y)$ is jointly continuous in $(t, x, y) \in(0, T] \times \mathbb{R}^{d} \times \mathbb{R}^{d}$. By (3.1) and induction method the same property holds for $q_{n}(t, x, y)$ for each $n \in N$. Since $\sum_{n=0}^{\infty} q_{n}(t, x, y)$ is absolutely and locally uniformly convergent we finally obtain that $q(t, x, y)$ is jointly continuous in $(t, x, y) \in(0, T] \times \mathbb{R}^{d} \times \mathbb{R}^{d}$.

By elementary calculations, for any $t>0, u, w \in \mathbb{R}$ satisfying $|u-w| \leq t^{1 / \alpha}$, we have

$$
\rho_{0}^{0}(t, u) \approx \rho_{0}^{0}(t, w)
$$


Lemma 3.3. There exists $c=c(\alpha, d)$ such that for any $m \in\{1, \ldots, d\}, t>0$, $x, x^{\prime} \in \mathbb{R}^{d}$ we have

$$
\left|\prod_{i=1}^{m} g_{t}\left(x_{i}\right)-\prod_{i=1}^{m} g_{t}\left(x_{i}^{\prime}\right)\right| \leq c\left(\prod_{i=1}^{m} g_{t}\left(\left|x_{i}\right| \wedge\left|x_{i}^{\prime}\right|\right)\right)\left[1 \wedge \sum_{j=1}^{m} \frac{\left|x_{j}-x_{j}^{\prime}\right|}{t^{1 / \alpha}+\left|x_{i}\right| \wedge\left|x_{i}^{\prime}\right|}\right] .
$$

If additionally $\left|x-x^{\prime}\right| \leq t^{1 / \alpha}$, then

$$
\left|\prod_{i=1}^{m} g_{t}\left(x_{i}\right)-\prod_{i=1}^{m} g_{t}\left(x_{i}^{\prime}\right)\right| \leq c t^{m}\left(\prod_{i=1}^{m} \rho_{0}^{0}\left(t, x_{i}\right)\right)\left[1 \wedge \sum_{j=1}^{m} t^{-1 / \alpha}\left|x_{j}-x_{j}^{\prime}\right|\right] .
$$

Proof: Let $g_{t}^{(3)}(\cdot)$ be the radial profile of the transition density of the standard 3 -dimensional $\alpha$-stable isotropic process. Then it is well known (see e.g. Bogdan and Jakubowski, 2007, (11)) that

$$
\frac{d g_{t}(x)}{d x}=-4 \pi x g_{t}^{(3)}(|x|), \quad x \in \mathbb{R} .
$$

By the standard estimates of transition density of the $\alpha$-stable isotropic process we have

which yields

$$
g_{t}^{(3)}(|x|) \leq c \frac{g_{t}(x)}{\left(|x|+t^{1 / \alpha}\right)^{2}}
$$

$$
\left|\frac{d g_{t}(x)}{d x}\right| \leq c \frac{|x|}{\left(|x|+t^{1 / \alpha}\right)^{2}} g_{t}(x) \leq c \frac{g_{t}(x)}{|x|+t^{1 / \alpha}}, \quad x \in \mathbb{R} .
$$

Next, for any $u, w \in \mathbb{R}$, from the above gradient estimate of $g_{t}$ and the fact that $g_{t}(u)$ is decreasing in $|u|$,

$$
\left|g_{t}(u)-g_{t}(w)\right| \leq c \frac{|u-w|}{|u| \wedge|w|+t^{1 / \alpha}} g_{t}(|u| \wedge|w|) .
$$

Hence, by monotonicity of $g_{t}(u)$ and (3.18),

$$
\begin{aligned}
& \left|\prod_{i=1}^{m} g_{t}\left(x_{i}\right)-\prod_{i=1}^{m} g_{t}\left(x_{i}^{\prime}\right)\right| \\
& \leq \sum_{i=1}^{m}\left[\left|g_{t}\left(x_{i}\right)-g_{t}\left(x_{i}^{\prime}\right)\right| \prod_{\substack{j \neq i, 1 \leq j \leq m \\
\text { s. }}} g_{t}\left(\left|x_{i}\right| \wedge\left|x_{i}^{\prime}\right|\right)\right] \\
& \leq c\left(\prod_{i=1}^{m} g_{t}\left(\left|x_{i}\right| \wedge\left|x_{i}^{\prime}\right|\right)\right) \sum_{j=1}^{m} \frac{\left|x_{i}-x_{i}^{\prime}\right|}{\left|x_{i}\right| \wedge\left|x_{i}^{\prime}\right|+t^{1 / \alpha}}
\end{aligned}
$$

Combining this with the obvious inequality

$$
\left|\prod_{i=1}^{m} g_{t}\left(x_{i}\right)-\prod_{i=1}^{m} g_{t}\left(x_{i}^{\prime}\right)\right| \leq \prod_{i=1}^{m} g_{t}\left(\left|x_{i}\right| \wedge\left|x_{i}^{\prime}\right|\right)
$$

we finish the proof of (3.16).

To get the second inequality we apply (3.15).

As a direct conclusion of Lemma 3.3 we get 
Corollary 3.4. For any $k \in\{1, \ldots, d\}, t>0, x, x^{\prime}, y \in \mathbb{R}^{d}$ satisfying $\left|x-x^{\prime}\right| \leq t^{1 / \alpha}$ we have

$$
\begin{aligned}
& \left|\prod_{\substack{i=1 \\
i \neq k}}^{d} a_{i i}(y) g_{t}\left(\frac{x_{i}}{a_{i i}(y)}\right)-\prod_{\substack{i=1 \\
i \neq k}}^{d} a_{i i}(y) g_{t}\left(\frac{x_{i}^{\prime}}{a_{i i}(y)}\right)\right| \\
& \leq c t^{d-1}\left(\prod_{\substack{i=1 \\
i \neq k}}^{d} \rho_{0}^{0}\left(t, x_{i}\right)\right) \sum_{j=1}^{d}\left(t^{-1 / \alpha}\left|x_{j}-x_{j}^{\prime}\right| \wedge 1\right) .
\end{aligned}
$$

Corollary 3.5. For any $t>0$ and $x, y, w \in \mathbb{R}^{d}$ we have

$$
\left|p_{x}(t, w)-p_{y}(t, w)\right| \leq c p_{x}(t, w)\left(|x-y|^{\beta} \wedge 1\right) .
$$

Proof: We have

$$
\begin{aligned}
\left|p_{x}(t, w)-p_{y}(t, w)\right| & =\left|\prod_{i=1}^{d} a_{i i}^{-1}(y) g_{t}\left(\frac{w_{i}}{a_{i i}(y)}\right)-\prod_{i=1}^{d} a_{i i}^{-1}(x) g_{t}\left(\frac{w_{i}}{a_{i i}(x)}\right)\right| \\
& \leq\left|\prod_{i=1}^{d} a_{i i}^{-1}(y)-\prod_{i=1}^{d} a_{i i}^{-1}(x)\right| \prod_{i=1}^{d} g_{t}\left(\frac{w_{i}}{a_{i i}(y)}\right) \\
& +\prod_{i=1}^{d} a_{i i}^{-1}(x)\left|\prod_{i=1}^{d} g_{t}\left(\frac{w_{i}}{a_{i i}(y)}\right)-\prod_{i=1}^{d} g_{t}\left(\frac{w_{i}}{a_{i i}(x)}\right)\right|
\end{aligned}
$$

Next, by (1.3) and (1.2),

$$
\left|\prod_{i=1}^{d} a_{i i}^{-1}(y)-\prod_{i=1}^{d} a_{i i}^{-1}(x)\right| \leq c\left(|x-y|^{\beta} \wedge 1\right),
$$

and by Lemma 3.3 together with (1.3) and (1.2)

$$
\begin{aligned}
& \left|\prod_{i=1}^{d} g_{t}\left(\frac{w_{i}}{a_{i i}(y)}\right)-\prod_{i=1}^{d} g_{t}\left(\frac{w_{i}}{a_{i i}(x)}\right)\right| \\
\leq & c\left[1 \wedge \sum_{j=1}^{m} \frac{\left|w_{i}\right|\left|a_{i i}(x)-a_{i i}(y)\right|}{t^{1 / \alpha}+\left|w_{i}\right|}\right] \prod_{i=1}^{d} g_{t}\left(\frac{w_{i}}{a_{i i}(x)}\right) \\
\leq & c\left(|x-y|^{\beta} \wedge 1\right) \prod_{i=1}^{d} g_{t}\left(\frac{w_{i}}{a_{i i}(x)}\right) .
\end{aligned}
$$

The proof is completed.

Corollary 3.6. For any $x \in \mathbb{R}^{d}, t>0$ we have

$$
\int_{\mathbb{R}^{d-1}}\left|\prod_{i=2}^{d} \frac{1}{a_{i i}(y)} g_{t}\left(\frac{x_{i}-y_{i}}{a_{i i}(y)}\right)-\prod_{i=2}^{d} \frac{1}{a_{i i}(\tilde{y})} g_{t}\left(\frac{x_{i}-y_{i}}{a_{i i}(\tilde{y})}\right)\right| d y_{2} \ldots d y_{d} \leq c\left(\left|x_{1}-y_{1}\right|^{\beta} \wedge 1\right),
$$

where $\tilde{y}=\left(x_{1}, y_{2}, \ldots, y_{d}\right)$. 
Proof: By the same arguments as in the proof Corollary 3.5 we have

$$
\left|\prod_{i=2}^{d} \frac{1}{a_{i i}(y)} g_{t}\left(\frac{x_{i}-y_{i}}{a_{i i}(y)}\right)-\prod_{i=2}^{d} \frac{1}{a_{i i}(\tilde{y})} g_{t}\left(\frac{x_{i}-y_{i}}{a_{i i}(\tilde{y})}\right)\right| \leq c\left[\prod_{i=2}^{d} g_{t}\left(x_{i}-y_{i}\right)\right]\left(|y-\tilde{y}|^{\beta} \wedge 1\right) .
$$

Observing that $|y-\tilde{y}|=\left|x_{1}-y_{1}\right|$ we obtain the conclusion by integration.

Lemma 3.7. For any $t \in(0, T], x, x^{\prime}, y \in \mathbb{R}^{d}$ satisfying $\left|x-x^{\prime}\right| \leq t^{1 / \alpha}$ we have

$$
\begin{aligned}
& \sum_{k=1}^{d} \int_{\mathbb{R}}\left|\delta_{p_{y}}\left(t, x, z_{k} e_{k}\right)-\delta_{p_{y}}\left(t, x^{\prime}, z_{k} e_{k}\right)\right| \frac{d z_{k}}{\left|z_{k}\right|^{1+\alpha}} \\
& \leq c t^{d-1}\left(\prod_{i=1}^{d} \rho_{0}^{0}\left(t, x_{i}\right)\right) \sum_{j=1}^{d}\left(t^{-1 / \alpha}\left|x_{j}-x_{j}^{\prime}\right| \wedge 1\right) .
\end{aligned}
$$

Proof: Fix $k \in\{1, \ldots, d\}$ For $t>0, z \in \mathbb{R}$ put

$$
h_{y}(t, z)=a_{k k}(y) g_{t}\left(\frac{z}{a_{k k}(y)}\right) \text {. }
$$

We have

$$
\begin{aligned}
& \int_{\mathbb{R}}\left|\delta_{p_{y}}\left(t, x, z_{k} e_{k}\right)-\delta_{p_{y}}\left(t, x^{\prime}, z_{k} e_{k}\right)\right| \frac{d z_{k}}{\left|z_{k}\right|^{1+\alpha}} \\
& =\int_{\mathbb{R}} \mid\left[\prod_{\substack{i=1 \\
i \neq k}}^{d} a_{i i}(y) g_{t}\left(\frac{x_{i}}{a_{i i}(y)}\right)\right] \delta_{h_{y}}\left(t, x_{k}, z_{k}\right) \\
& -\left[\prod_{\substack{i=1 \\
i \neq k}}^{d} a_{i i}(y) g_{t}\left(\frac{x_{i}^{\prime}}{a_{i i}(y)}\right)\right] \delta_{h_{y}}\left(t, x_{k}^{\prime}, z_{k}\right) \mid \frac{d z_{k}}{\left|z_{k}\right|^{1+\alpha}} \\
& \leq\left|\prod_{\substack{i=1 \\
i \neq k}}^{d} a_{i i}(y) g_{t}\left(\frac{x_{i}}{a_{i i}(y)}\right)-\prod_{\substack{i=1 \\
i \neq k}}^{d} a_{i i}(y) g_{t}\left(\frac{x_{i}^{\prime}}{a_{i i}(y)}\right)\right| \int_{\mathbb{R}}\left|\delta_{h_{y}}\left(t, x_{k}, z_{k}\right)\right| \frac{d z_{k}}{\left|z_{k}\right|^{1+\alpha}} \\
& +\left[\prod_{\substack{i=1 \\
i \neq k}}^{d} a_{i i}(y) g_{t}\left(\frac{x_{i}^{\prime}}{a_{i i}(y)}\right)\right] \int_{\mathbb{R}}\left|\delta_{h_{y}}\left(t, x_{k}, z_{k}\right)-\delta_{h_{y}}\left(t, x_{k}^{\prime}, z_{k}\right)\right| \frac{d z_{k}}{\left|z_{k}\right|^{1+\alpha}} .
\end{aligned}
$$

By Chen and Zhang (2016, (2.28)) we have

$$
\int_{\mathbb{R}}\left|\delta_{h_{y}}\left(t, x_{k}, z_{k}\right)\right| \frac{d z_{k}}{\left|z_{k}\right|^{1+\alpha}} \leq c \rho_{0}^{0}\left(t, x_{k}\right)
$$

while, by Chen and Zhang (2016, (2.29)),

$$
\int_{\mathbb{R}}\left|\delta_{h_{y}}\left(t, x_{k}, z_{k}\right)-\delta_{h_{y}}\left(t, x_{k}^{\prime}, z_{k}\right)\right| \frac{d z_{k}}{\left|z_{k}\right|^{1+\alpha}} \leq c \rho_{0}^{0}\left(t, x_{k}\right)\left(t^{-1 / \alpha}\left|x_{k}-x_{k}^{\prime}\right| \wedge 1\right) .
$$

Applying the above inequalities and Corollary 3.4 we obtain the desired bound (3.20). 
Lemma 3.8. For any $x, x^{\prime}, y \in \mathbb{R}^{d}, t \in(0, T]$ and $\gamma \in(0, \beta)$ we have

$$
\begin{aligned}
& \left.\left|q_{0}(t, x, y)-q_{0}\left(t, x^{\prime}, y\right)\right| \leq c\left(\left|x-x^{\prime}\right|^{\beta-\gamma} \wedge 1\right)\right]\left(\rho_{\gamma}^{0}\left(t, x_{k}-y_{k}\right)+\rho_{\gamma-\beta}^{\beta}\left(t, x_{k}-y_{k}\right)\right) \\
& \times\left[\sum_{k=1}^{d}\left[\prod_{\substack{i=1 \\
i \neq k}}^{d}\left(t \rho_{0}^{0}\left(t, x_{i}-y_{i}\right)\right)\right]\right. \\
& \left.+\sum_{k=1}^{d}\left[\prod_{\substack{i=1 \\
i \neq k}}^{d}\left(t \rho_{0}^{0}\left(t, x_{i}^{\prime}-y_{i}\right)\right)\right]\left(\rho_{\gamma}^{0}\left(t, x_{k}^{\prime}-y_{k}\right)+\rho_{\gamma-\beta}^{\beta}\left(t, x_{k}^{\prime}-y_{k}\right)\right)\right]
\end{aligned}
$$

Proof: Case 1. $\left|x-x^{\prime}\right|>t^{1 / \alpha}$.

By (3.5) we get, for $z=x$ or $x^{\prime}$,

$$
\left|q_{0}(t, z, y)\right| \leq c\left(\left|x-x^{\prime}\right|^{\beta-\gamma} \wedge 1\right) t^{d-1}\left(\prod_{i=1}^{d} \rho_{0}^{0}\left(t, z_{i}-y_{i}\right)\right)\left(\sum_{m=1}^{d}\left(\left|z_{m}-y_{m}\right| \wedge 1\right)^{\beta} t^{\frac{\gamma-\beta}{\alpha}}\right) .
$$

Case 2. $\left|x-x^{\prime}\right| \leq t^{1 / \alpha}$.

We have

$$
\begin{aligned}
& \left|q_{0}(t, x, y)-q_{0}\left(t, x^{\prime}, y\right)\right| \\
& =\frac{\mathcal{A}}{2} \mid \sum_{k=1}^{d} \int_{\mathbb{R}} \delta_{p_{y}}\left(t, x-y, z_{k} e_{k}\right)\left(a_{k k}^{\alpha}(x)-a_{k k}^{\alpha}(y)\right) \frac{d z_{k}}{\left|z_{k}\right|^{1+\alpha}} \\
& -\sum_{k=1}^{d} \int_{\mathbb{R}} \delta_{p_{y}}\left(t, x^{\prime}-y, z_{k} e_{k}\right)\left(a_{k k}^{\alpha}\left(x^{\prime}\right)-a_{k k}^{\alpha}(y)\right) \frac{d z_{k}}{\left|z_{k}\right|^{\mid+\alpha} \mid} \\
& \leq \sum_{k=1}^{d} \int_{\mathbb{R}}\left|\delta_{p_{y}}\left(t, x-y, z_{k} e_{k}\right)-\delta_{p_{y}}\left(t, x^{\prime}-y, z_{k} e_{k}\right)\right|\left|a_{k k}^{\alpha}(x)-a_{k k}^{\alpha}(y)\right| \frac{d z_{k}}{\left|z_{k}\right|^{1+\alpha}} \\
& +\sum_{k=1}^{d} \int_{\mathbb{R}}\left|\delta_{p_{y}}\left(t, x^{\prime}-y, z_{k} e_{k}\right)\right|\left|a_{k k}^{\alpha}(x)-a_{k k}^{\alpha}\left(x^{\prime}\right)\right| \frac{d z_{k}}{\left|z_{k}\right|^{1+\alpha}} \\
& \leq c\left(|x-y|^{\beta} \wedge 1\right) \sum_{k=1}^{d} \int_{\mathbb{R}}\left|\delta_{p_{y}}\left(t, x-y, z_{k} e_{k}\right)-\delta_{p_{y}}\left(t, x^{\prime}-y, z_{k} e_{k}\right)\right| \frac{d z_{k}}{\left|z_{k}\right|^{1+\alpha}} \\
& +c\left(\left|x-x^{\prime}\right|^{\beta} \wedge 1\right) \sum_{k=1}^{d} \int_{\mathbb{R}}\left|\delta_{p_{y}}\left(t, x^{\prime}-y, z_{k} e_{k}\right)\right| \frac{d z_{k}}{\left|z_{k}\right|^{1+\alpha}} \\
& =\mathrm{I}+\mathrm{II} .
\end{aligned}
$$

By Lemma 3.7 we get

$$
\mathrm{I} \leq c\left(|x-y|^{\beta} \wedge 1\right)\left(t^{-1 / \alpha}\left|x-x^{\prime}\right| \wedge 1\right) t^{d-1} \prod_{i=1}^{d} \rho_{0}^{0}\left(t, x_{i}-y_{i}\right) .
$$

Note that $t^{-1 / \alpha}\left|x-x^{\prime}\right| \leq 1$ so $t^{-1 / \alpha}\left|x-x^{\prime}\right| \leq t^{\frac{\gamma-\beta}{\alpha}}\left|x-x^{\prime}\right|^{\beta-\gamma}$. Let $m \in\{1, \ldots, d\}$ be such that $\left|x_{m}-y_{m}\right|=\max _{i \in\{1, \ldots, d\}}\left|x_{i}-y_{i}\right|$. It follows that (3.21) is bounded from above by 


$$
\begin{aligned}
& c t^{\frac{\gamma-\beta}{\alpha}}\left|x-x^{\prime}\right|^{\beta-\gamma} t^{d-1}\left(\prod_{i=1}^{d} \rho_{0}^{0}\left(t, x_{i}-y_{i}\right)\right)\left(\left|x_{m}-y_{m}\right|^{\beta} \wedge 1\right) \\
& \leq c\left(\left|x-x^{\prime}\right|^{\beta-\gamma} \wedge 1\right)\left(\prod_{\substack{i=1 \\
i \neq m}}^{d} \rho_{0}^{0}\left(t, x_{i}-y_{i}\right)\right) \rho_{\gamma-\beta}^{\beta}\left(t, x_{m}-y_{m}\right) .
\end{aligned}
$$

We have $\left|x-x^{\prime}\right| \leq t^{1 / \alpha}$ so $1 \leq\left|x-x^{\prime}\right|^{-\gamma} t^{\gamma / \alpha}$. It follows that $\left(\left|x-x^{\prime}\right|^{\beta} \wedge 1\right) \leq$ $\left(\left|x-x^{\prime}\right|^{\beta-\gamma} \wedge 1\right) t^{\gamma / \alpha}$. Using this and Lemma 3.1 we get

$$
\begin{aligned}
\mathrm{II} & \leq c\left(\left|x-x^{\prime}\right|^{\beta} \wedge 1\right) t^{d-1} \prod_{i=1}^{d} \rho_{0}^{0}\left(t, x_{i}^{\prime}-y_{i}\right) \\
& \leq c\left(\left|x-x^{\prime}\right|^{\beta-\gamma} \wedge 1\right) t^{d-1} \sum_{k=1}^{d}\left(\prod_{\substack{i=1 \\
i \neq k}}^{d} \rho_{0}^{0}\left(t, x_{i}^{\prime}-y_{i}\right)\right) \rho_{\gamma}^{0}\left(t, x_{k}^{\prime}-y_{k}\right) .
\end{aligned}
$$

Theorem 3.9. For any $x, x^{\prime}, y \in \mathbb{R}^{d}, t \in(0, T]$ and $\gamma \in(0, \beta)$ we have

$$
\begin{aligned}
& \left|q(t, x, y)-q\left(t, x^{\prime}, y\right)\right| \leq c\left(\left|x-x^{\prime}\right|^{\beta-\gamma} \wedge 1\right) \\
& \times\left[\sum_{k=1}^{d}\left[\prod_{\substack{i=1 \\
i \neq k}}^{d}\left(t \rho_{0}^{0}\left(t, x_{i}-y_{i}\right)\right)\right]\left(\rho_{\gamma}^{0}\left(t, x_{k}-y_{k}\right)+\rho_{\gamma-\beta}^{\beta}\left(t, x_{k}-y_{k}\right)\right)\right. \\
& \left.+\sum_{k=1}^{d}\left[\prod_{\substack{i=1 \\
i \neq k}}^{d}\left(t \rho_{0}^{0}\left(t, x_{i}^{\prime}-y_{i}\right)\right)\right]\left(\rho_{\gamma}^{0}\left(t, x_{k}^{\prime}-y_{k}\right)+\rho_{\gamma-\beta}^{\beta}\left(t, x_{k}^{\prime}-y_{k}\right)\right)\right] .
\end{aligned}
$$

Proof: By the definition of $q_{n},(3.14)$ and Lemma 3.8 we get for $n \in \mathbb{N}$

$$
\begin{aligned}
& \left|q_{n}(t, x, y)-q_{n}\left(t, x^{\prime}, y\right)\right| \\
& \leq \int_{0}^{t} \int_{\mathbb{R}^{d}}\left|q_{0}(t-s, x, z)-q_{0}\left(t-s, x^{\prime}, z\right)\right|\left|q_{n-1}(s, z, y)\right| d z d s \\
& \leq \frac{c C^{n-1}}{(n !)^{\beta / \alpha}}\left(\left|x-x^{\prime}\right|^{\beta-\gamma} \wedge 1\right)\left(A(t, x, y)+A\left(t, x^{\prime}, y\right)\right),
\end{aligned}
$$

where $C$ is the constant from (3.14) and

$$
\begin{aligned}
& A(t, x, y) \\
& =\int_{0}^{t} \int_{\mathbb{R}^{d}} \sum_{k=1}^{d}\left[\prod_{\substack{i=1 \\
i \neq k}}^{d} \rho_{\alpha}^{0}\left(t-s, x_{i}-z_{i}\right)\right]\left(\rho_{\gamma}^{0}\left(t-s, x_{k}-z_{k}\right)+\rho_{\gamma-\beta}^{\beta}\left(t-s, x_{k}-z_{k}\right)\right) \\
& \times \sum_{m=1}^{d}\left[\prod_{\substack{j=1 \\
j \neq m}}^{d} \rho_{\alpha}^{0}\left(s, z_{j}-y_{j}\right)\right]\left(\rho_{\beta}^{0}\left(s, z_{m}-y_{m}\right)+\rho_{0}^{\beta}\left(s, z_{m}-y_{m}\right)\right) d z_{1} \ldots d z_{d} d s .
\end{aligned}
$$


We have

$$
\begin{aligned}
A(t, x, y)= & \sum_{k=1}^{d}\left[\prod_{\substack{i=1 \\
i \neq k}}^{d} t \rho_{0}^{0}\left(t, x_{i}-y_{i}\right)\right] B_{k}(t, x, y) \\
& +\sum_{k=1}^{d} \sum_{\substack{m=1 \\
m \neq k}}^{d}\left[\prod_{\substack{i=1 \\
i \neq k, i \neq m}}^{d} t \rho_{0}^{0}\left(t, x_{i}-y_{i}\right)\right] \\
& \times\left[D_{k, m}(t, x, y)+E_{k, m}(t, x, y)+F_{k, m}(t, x, y)+G_{k, m}(t, x, y) \beta, 24\right)
\end{aligned}
$$

where

$$
\begin{aligned}
B_{k}(t, x, y)= & \int_{0}^{t} \int_{\mathbb{R}}\left(\rho_{\gamma}^{0}\left(t-s, x_{k}-z_{k}\right)+\rho_{\gamma-\beta}^{\beta}\left(t-s, x_{k}-z_{k}\right)\right) \\
& \times\left(\rho_{\beta}^{0}\left(s, z_{k}-y_{k}\right)+\rho_{0}^{\beta}\left(s, z_{k}-y_{k}\right)\right) d z_{k} d s \\
D_{k, m}(t, x, y)= & \int_{0}^{t} \int_{\mathbb{R}} \rho_{\gamma}^{0}\left(t-s, x_{k}-z_{k}\right) \rho_{\alpha}^{0}\left(s, z_{k}-y_{k}\right) d z_{k} \\
& \times \int_{\mathbb{R}} \rho_{\alpha}^{0}\left(t-s, x_{m}-z_{m}\right) \rho_{\beta}^{0}\left(s, z_{m}-y_{m}\right) d z_{m} d s, \\
E_{k, m}(t, x, y)= & \int_{0}^{t} \int_{\mathbb{R}} \rho_{\gamma}^{0}\left(t-s, x_{k}-z_{k}\right) \rho_{\alpha}^{0}\left(s, z_{k}-y_{k}\right) d z_{k} \\
& \times \int_{\mathbb{R}} \rho_{\alpha}^{0}\left(t-s, x_{m}-z_{m}\right) \rho_{0}^{\beta}\left(s, z_{m}-y_{m}\right) d z_{m} d s, \\
F_{k, m}(t, x, y)= & \int_{0}^{t} \int_{\mathbb{R}} \rho_{\gamma-\beta}^{\beta}\left(t-s, x_{k}-z_{k}\right) \rho_{\alpha}^{0}\left(s, z_{k}-y_{k}\right) d z_{k} \\
& \times \int_{\mathbb{R}} \rho_{\alpha}^{0}\left(t-s, x_{m}-z_{m}\right) \rho_{\beta}^{0}\left(s, z_{m}-y_{m}\right) d z_{m} d s, \\
& \int_{0}^{t} \int_{\mathbb{R}} \rho_{\gamma-\beta}^{\beta}\left(t-s, x_{k}-z_{k}\right) \rho_{\alpha}^{0}\left(s, z_{k}-y_{k}\right) d z_{k} \\
& \times \int_{\mathbb{R}} \rho_{\alpha}^{0}\left(t-s, x_{m}-z_{m}\right) \rho_{0}^{\beta}\left(s, z_{m}-y_{m}\right) d z_{m} d s . \\
G_{k, m}(t, x, y) &
\end{aligned}
$$

By (2.5) we get

$$
B_{k}(t, x, y) \leq c\left(\rho_{\gamma}^{0}\left(t, x_{k}-y_{k}\right)+\rho_{\gamma-\beta}^{\beta}\left(t, x_{k}-y_{k}\right)\right) .
$$

Using (2.4) we obtain

$$
\begin{aligned}
& D_{k, m}(t, x, y) \\
& \leq c \int_{0}^{t}\left((t-s)^{\frac{\gamma-\alpha}{\alpha}} s+(t-s)^{\frac{\gamma}{\alpha}}\right)\left(s^{\frac{\beta}{\alpha}}+(t-s) s^{\frac{\beta-\alpha}{\alpha}}\right) d s \rho_{0}^{0}\left(t, x_{k}-y_{k}\right) \rho_{0}^{0}\left(t, x_{m}-y_{m}\right) \\
& \leq c t^{1+\frac{\gamma}{\alpha}+\frac{\beta}{\alpha}} \rho_{0}^{0}\left(t, x_{k}-y_{k}\right) \rho_{0}^{0}\left(t, x_{m}-y_{m}\right) \\
& \leq c t \rho_{\gamma}^{0}\left(t, x_{k}-y_{k}\right) \rho_{0}^{0}\left(t, x_{m}-y_{m}\right)
\end{aligned}
$$




$$
\begin{aligned}
& E_{k, m}(t, x, y) \\
& \leq c \int_{0}^{t}\left((t-s)^{\frac{\gamma-\alpha}{\alpha}} s+(t-s)^{\frac{\gamma}{\alpha}}\right)\left((t-s)^{\beta / \alpha}+(t-s) s^{\frac{\beta-\alpha}{\alpha}}\right) d s \\
& \times \rho_{0}^{0}\left(t, x_{k}-y_{k}\right) \rho_{0}^{0}\left(t, x_{m}-y_{m}\right) \\
& +c \int_{0}^{t}\left((t-s)^{\frac{\gamma-\alpha}{\alpha}} s+(t-s)^{\frac{\gamma}{\alpha}}\right) d s \rho_{0}^{0}\left(t, x_{k}-y_{k}\right) \rho_{0}^{\beta}\left(t, x_{m}-y_{m}\right) \\
& \leq c t^{1+\frac{\gamma}{\alpha}+\frac{\beta}{\alpha}} \rho_{0}^{0}\left(t, x_{k}-y_{k}\right) \rho_{0}^{0}\left(t, x_{m}-y_{m}\right)+c t^{1+\frac{\gamma}{\alpha}} \rho_{0}^{0}\left(t, x_{k}-y_{k}\right) \rho_{0}^{\beta}\left(t, x_{m}-y_{m}\right) \\
& \leq \operatorname{ct} \rho_{\gamma}^{0}\left(t, x_{k}-y_{k}\right) \rho_{0}^{0}\left(t, x_{m}-y_{m}\right) \text {, } \\
& F_{k, m}(t, x, y) \\
& \leq c \int_{0}^{t}\left((t-s)^{\frac{\gamma-\alpha}{\alpha}} s+(t-s)^{\frac{\gamma-\beta}{\alpha}} s^{\frac{\beta}{\alpha}}\right)\left(s^{\frac{\beta}{\alpha}}+(t-s) s^{\frac{\beta-\alpha}{\alpha}}\right) d s \\
& \times \rho_{0}^{0}\left(t, x_{k}-y_{k}\right) \rho_{0}^{0}\left(t, x_{m}-y_{m}\right) \\
& +c \int_{0}^{t}\left((t-s)^{\frac{\gamma-\beta}{\alpha}}\left(s^{\frac{\beta}{\alpha}}+(t-s) s^{\frac{\beta-\alpha}{\alpha}}\right)\right) d s \rho_{0}^{\beta}\left(t, x_{k}-y_{k}\right) \rho_{0}^{0}\left(t, x_{m}-y_{m}\right) \\
& \leq c t^{1+\frac{\gamma}{\alpha}+\frac{\beta}{\alpha}} \rho_{0}^{0}\left(t, x_{k}-y_{k}\right) \rho_{0}^{0}\left(t, x_{m}-y_{m}\right)+c t^{1+\frac{\gamma}{\alpha}} \rho_{0}^{\beta}\left(t, x_{k}-y_{k}\right) \rho_{0}^{0}\left(t, x_{m}-y_{m}\right) \\
& \leq \operatorname{ct} \rho_{\gamma}^{0}\left(t, x_{k}-y_{k}\right) \rho_{0}^{0}\left(t, x_{m}-y_{m}\right) \text {, } \\
& G_{k, m}(t, x, y) \\
& \leq c \int_{0}^{t}\left((t-s)^{\frac{\gamma-\alpha}{\alpha}} s+(t-s)^{\frac{\gamma-\beta}{\alpha}} s^{\frac{\beta}{\alpha}}\right)\left((t-s)^{\frac{\beta}{\alpha}}+(t-s) s^{\frac{\beta-\alpha}{\alpha}}\right) d s \\
& \times \rho_{0}^{0}\left(t, x_{k}-y_{k}\right) \rho_{0}^{0}\left(t, x_{m}-y_{m}\right) \\
& +c \int_{0}^{t}\left((t-s)^{\frac{\gamma-\alpha}{\alpha}} s+(t-s)^{\frac{\gamma-\beta}{\alpha}} s^{\frac{\beta}{\alpha}}\right) d s \rho_{0}^{0}\left(t, x_{k}-y_{k}\right) \rho_{0}^{\beta}\left(t, x_{m}-y_{m}\right) \\
& +c \int_{0}^{t}\left((t-s)^{\frac{\gamma-\beta}{\alpha}}\left((t-s)^{\frac{\beta}{\alpha}}+(t-s) s^{\frac{\beta-\alpha}{\alpha}}\right)\right) d s \rho_{0}^{\beta}\left(t, x_{k}-y_{k}\right) \rho_{0}^{0}\left(t, x_{m}-y_{m}\right) \\
& +c \int_{0}^{t}(t-s)^{\frac{\gamma-\beta}{\alpha}} d s \rho_{0}^{\beta}\left(t, x_{k}-y_{k}\right) \rho_{0}^{\beta}\left(t, x_{m}-y_{m}\right) \\
& \leq c t^{1+\frac{\gamma}{\alpha}+\frac{\beta}{\alpha}} \rho_{0}^{0}\left(t, x_{k}-y_{k}\right) \rho_{0}^{0}\left(t, x_{m}-y_{m}\right)+c t^{1+\frac{\gamma}{\alpha}} \rho_{0}^{0}\left(t, x_{k}-y_{k}\right) \rho_{0}^{\beta}\left(t, x_{m}-y_{m}\right) \\
& +c t^{1+\frac{\gamma}{\alpha}} \rho_{0}^{\beta}\left(t, x_{k}-y_{k}\right) \rho_{0}^{0}\left(t, x_{m}-y_{m}\right)+c t^{1+\frac{\gamma}{\alpha}-\frac{\beta}{\alpha}} \rho_{0}^{\beta}\left(t, x_{k}-y_{k}\right) \rho_{0}^{\beta}\left(t, x_{m}-y_{m}\right) \\
& \leq c t \rho_{\gamma}^{0}\left(t, x_{k}-y_{k}\right) \rho_{0}^{0}\left(t, x_{m}-y_{m}\right)+\operatorname{ct} \rho_{\gamma-\beta}^{\beta}\left(t, x_{k}-y_{k}\right) \rho_{0}^{0}\left(t, x_{m}-y_{m}\right) \text {. }
\end{aligned}
$$

By (3.24-3.29) we obtain

$$
A(t, x, y) \leq c \sum_{k=1}^{d}\left(\prod_{\substack{i=1 \\ i \neq k}}^{d} t \rho_{0}^{0}\left(t, x_{i}-y_{i}\right)\right)\left(\rho_{\gamma}^{0}\left(t, x_{k}-y_{k}\right)+\rho_{\gamma-\beta}^{\beta}\left(t, x_{k}-y_{k}\right)\right) .
$$

Using this and (3.23) we obtain that for any $n \in \mathbb{N}, x, x^{\prime}, y \in \mathbb{R}^{d}, t \in(0, T]$ and $\gamma \in(0, \beta)$

$$
\left|q_{n}(t, x, y)-q_{n}\left(t, x^{\prime}, y\right)\right| \leq \frac{c C^{n-1}}{(n !)^{\beta / \alpha}}\left(\left|x-x^{\prime}\right|^{\beta-\gamma} \wedge 1\right)
$$




$$
\begin{aligned}
& \times\left[\sum_{k=1}^{d}\left[\prod_{\substack{i=1 \\
i \neq k}}^{d}\left(t \rho_{0}^{0}\left(t, x_{i}-y_{i}\right)\right)\right]\left(\rho_{\gamma}^{0}\left(t, x_{k}-y_{k}\right)+\rho_{\gamma-\beta}^{\beta}\left(t, x_{k}-y_{k}\right)\right)\right. \\
& \left.+\sum_{k=1}^{d}\left[\prod_{\substack{i=1 \\
i \neq k}}^{d}\left(t \rho_{0}^{0}\left(t, x_{i}^{\prime}-y_{i}\right)\right)\right]\left(\rho_{\gamma}^{0}\left(t, x_{k}^{\prime}-y_{k}\right)+\rho_{\gamma-\beta}^{\beta}\left(t, x_{k}^{\prime}-y_{k}\right)\right)\right] .
\end{aligned}
$$

This, Lemma 3.8 and the definition of $q$ imply the assertion of the theorem.

Lemma 3.10. For all $\gamma \in(0,1], x, x^{\prime}, y \in \mathbb{R}^{d}, t>0$, we have

$$
\left|p_{y}(t, x)-p_{y}\left(t, x^{\prime}\right)\right| \leq c\left|x-x^{\prime}\right|^{\gamma} t^{-\gamma / \alpha}\left(\left[\prod_{i=1}^{d} g_{t}\left(x_{i}\right)\right]+\left[\prod_{i=1}^{d} g_{t}\left(x_{i}^{\prime}\right)\right]\right) .
$$

Proof: By Lemma 3.3 we get

$$
\left|p_{y}(t, x)-p_{y}\left(t, x^{\prime}\right)\right| \leq c\left(\left|x-x^{\prime}\right| t^{-1 / \alpha} \wedge 1\right)\left(\left[\prod_{i=1}^{d} g_{t}\left(x_{i}\right)\right]+\left[\prod_{i=1}^{d} g_{t}\left(x_{i}^{\prime}\right)\right]\right) .
$$

Since $\left(\left|x-x^{\prime}\right| t^{-1 / \alpha} \wedge 1\right) \leq\left|x-x^{\prime}\right|^{\gamma} t^{-\gamma / \alpha}$ we obtain the assertion of the lemma.

By Lemma 3.3 and the formula for $p_{y}(t, x)$ we obtain

Lemma 3.11. For any $x, y \in \mathbb{R}^{d}$ and $t>0$ we have

$$
\left|\nabla p_{y}(t, \cdot)(x-y)\right| \leq c t^{-\frac{1}{\alpha}} \prod_{i=1}^{d} \rho_{\alpha}^{0}\left(t, x_{i}-y_{i}\right) .
$$

Lemma 3.12. For any $x \in \mathbb{R}^{d}$ and $t \in(0, T]$ we have

$$
\left|\int_{\mathbb{R}^{d}} \nabla p_{y}(t, \cdot)(x-y) d y\right| \leq c t^{\frac{\beta-1}{\alpha}} .
$$

Proof: Let $D_{1} p_{y}(t, \cdot)(x-y)=\lim _{h \rightarrow 0}\left(p_{y}\left(t, x-y+h e_{1}\right)-p_{y}(t, x-y)\right) / h$. It is enough to prove the estimate for $\mathrm{I}=\int_{\mathbb{R}^{d}} D_{1} p_{y}(t, \cdot)(x-y) d y$. Let $\gamma \in(0, \beta)$ and put $\tilde{y}=\left(x_{1}, y_{2}, \ldots, y_{d}\right)$. We have

$$
\begin{aligned}
|\mathrm{I}|= & \mid \int_{\mathbb{R}^{d}}\left[\frac{1}{a_{11}^{2}(y)} g_{t}^{\prime}\left(\frac{x_{1}-y_{1}}{a_{11}(y)}\right)\left[\prod_{i=2}^{d} \frac{1}{a_{i i}(y)} g_{t}\left(\frac{x_{i}-y_{i}}{a_{i i}(y)}\right)\right]\right. \\
& \left.-\frac{1}{a_{11}^{2}(\tilde{y})} g_{t}^{\prime}\left(\frac{x_{1}-y_{1}}{a_{11}(\tilde{y})}\right)\left[\prod_{i=2}^{d} \frac{1}{a_{i i}(\tilde{y})} g_{t}\left(\frac{x_{i}-y_{i}}{a_{i i}(\tilde{y})}\right)\right]\right] d y \mid \\
\leq & \mid \int_{\mathbb{R}^{d-1}}\left[\int_{\mathbb{R}}\left[\frac{1}{a_{11}^{2}(y)} g_{t}^{\prime}\left(\frac{x_{1}-y_{1}}{a_{11}(y)}\right)-\frac{1}{a_{11}^{2}(\tilde{y})} g_{t}^{\prime}\left(\frac{x_{1}-y_{1}}{a_{11}(\tilde{y})}\right)\right] d y_{1}\right] \\
& \times\left[\prod_{i=2}^{d} \frac{1}{a_{i i}(\tilde{y})} g_{t}\left(\frac{x_{i}-y_{i}}{a_{i i}(\tilde{y})}\right)\right] d y_{2}, \ldots d y_{d} \mid \\
+ & \mid \int_{\mathbb{R}^{d}} \frac{1}{a_{11}^{2}(y)} g_{t}^{\prime}\left(\frac{x_{1}-y_{1}}{a_{11}(y)}\right) \\
& \times\left[\prod_{i=2}^{d} \frac{1}{a_{i i}(y)} g_{t}\left(\frac{x_{i}-y_{i}}{a_{i i}(y)}\right)-\prod_{i=2}^{d} \frac{1}{a_{i i}(\tilde{y})} g_{t}\left(\frac{x_{i}-y_{i}}{a_{i i}(\tilde{y})}\right)\right] d y \mid
\end{aligned}
$$




$$
=\mathrm{II}+\mathrm{III} \text {. }
$$

By Chen and Zhang (2016, (2.31)) we get

$$
\begin{aligned}
& \left|\frac{1}{a_{11}^{2}(y)} g_{t}^{\prime}\left(\frac{x_{1}-y_{1}}{a_{11}(y)}\right)-\frac{1}{a_{11}^{2}(\tilde{y})} g_{t}^{\prime}\left(\frac{x_{1}-y_{1}}{a_{11}(\tilde{y})}\right)\right| \\
& \leq c\left(\left|x_{1}-y_{1}\right|^{\beta} \wedge 1\right) t^{-1 / \alpha}\left(\rho_{\alpha}^{0}\left(t, x_{1}-y_{1}\right)+\rho_{\alpha-\gamma}^{\gamma}\left(t, x_{1}-y_{1}\right)\right) .
\end{aligned}
$$

Using this and (2.3) we obtain II $\leq c t^{\frac{\beta-1}{\alpha}}$. Note also that

$$
\left|\frac{1}{a_{11}^{2}(y)} g_{t}^{\prime}\left(\frac{x_{1}-y_{1}}{a_{11}(y)}\right)\right| \leq c \rho_{\alpha-1}^{0}\left(t, x_{1}-y_{1}\right) .
$$

Using this, Corollary 3.6 and (2.3) we get III $\leq c t^{\frac{\beta-1}{\alpha}}$.

Similarly as in Chen and Zhang (2016) we denote

$$
\phi_{y}(t, x, s)=\int_{\mathbb{R}^{d}} p_{z}(t-s, x-z) q(s, z, y) d z
$$

and

Clearly we have

$$
\varphi_{y}(t, x)=\int_{0}^{t} \phi_{y}(t, x, s) d s
$$

$$
p^{A}(t, x, y)=p_{y}(t, x-y)+\varphi_{y}(t, x) .
$$

By well known estimates of $\nabla p_{z}(t-s, \cdot)(x-z)$ and Theorem 3.2 we easily obtain the following result.

Lemma 3.13. For any $x, y \in \mathbb{R}^{d}, t>0$ and $s \in(0, t)$ we have

$$
\nabla_{x} \phi_{y}(t, x, s)=\int_{\mathbb{R}^{d}} \nabla p_{z}(t-s, \cdot)(x-z) q(s, z, y) d z .
$$

The next result is the most important step in proving gradient estimates of $p^{A}(t, x, y)$.

Lemma 3.14. For any $\alpha \in(1,2), x, y \in \mathbb{R}^{d}$ and $t \in(0, T]$ we have

$$
\nabla_{x} \varphi_{y}(t, x)=\int_{0}^{t} \int_{\mathbb{R}^{d}} \nabla p_{z}(t-s, \cdot)(x-z) q(s, z, y) d z d s .
$$

and

$$
\left|\nabla_{x} \varphi_{y}(t, x)\right| \leq c t^{-\frac{1}{\alpha}} \prod_{i=1}^{d} \rho_{\alpha}^{0}\left(t, x_{i}-y_{i}\right) .
$$

Proof: Let $x, y \in \mathbb{R}^{d}, t \in(0, T]$ and $s \in(0, t)$. The main tool used in this case is Theorem 3.2. Using this theorem, Lemmas 3.11, 3.13 and (2.4) we obtain

$$
\begin{aligned}
& \left|\nabla_{x} \phi_{y}(t, x, s)\right| \leq \int_{\mathbb{R}^{d}}\left|\nabla p_{z}(t-s, \cdot)(x-z)\right||q(s, z, y)| d z \\
& \leq c \int_{\mathbb{R}^{d}}(t-s)^{-1 / \alpha} p_{z}(t-s, x-z) \\
& \times \sum_{m=1}^{d}\left[\prod_{\substack{i=1 \\
i \neq m}}^{d} \rho_{\alpha}^{0}\left(s, z_{i}-y_{i}\right)\right]\left[\rho_{0}^{\beta}\left(s, z_{m}-y_{m}\right)+\rho_{\beta}^{0}\left(s, z_{m}-y_{m}\right)\right] d z
\end{aligned}
$$




$$
\begin{aligned}
& \leq c \sum_{m=1}^{d}\left[\prod_{\substack{i=1 \\
i \neq m}}^{d} \rho_{\alpha}^{0}\left(t, x_{i}-y_{i}\right)\right] \int_{\mathbb{R}} \rho_{\alpha-1}^{0}\left(t-s, x_{m}-z_{m}\right) \\
& \times\left[\rho_{0}^{\beta}\left(s, z_{m}-y_{m}\right)+\rho_{\beta}^{0}\left(s, z_{m}-y_{m}\right)\right] d z \\
& \leq c \sum_{m=1}^{d}\left[\prod_{\substack{i=1 \\
i \neq m}}^{d} \rho_{\alpha}^{0}\left(t, x_{i}-y_{i}\right)\right]\left[(t-s)^{\frac{\beta-1}{\alpha}} \rho_{0}^{0}\left(t, x_{m}-y_{m}\right)\right. \\
& +(t-s)^{\frac{\alpha-1}{\alpha}} s^{\frac{\beta-\alpha}{\alpha}} \rho_{0}^{0}\left(t, x_{m}-y_{m}\right)+(t-s)^{-\frac{1}{\alpha}} \rho_{0}^{\beta}\left(t, x_{m}-y_{m}\right) \\
& \left.+(t-s)^{-\frac{1}{\alpha}} s^{\frac{\beta}{\alpha}} \rho_{0}^{0}\left(t, x_{m}-y_{m}\right)\right] .
\end{aligned}
$$

It follows that

$$
\nabla_{x}\left[\int_{0}^{t} \phi_{y}(t, x, s) d s\right]=\int_{0}^{t} \nabla_{x} \phi_{y}(t, x, s) d s,
$$

which implies (3.31). We also obtain

$$
\begin{aligned}
& \left|\int_{0}^{t} \nabla_{x} \phi_{y}(t, x, s) d s\right| \\
& \leq c \sum_{m=1}^{d}\left[\prod_{\substack{i=1 \\
i \neq m}}^{d} \rho_{\alpha}^{0}\left(t, x_{i}-y_{i}\right)\right] \int_{0}^{t}\left[(t-s)^{\frac{\beta-1}{\alpha}} \rho_{0}^{0}\left(t, x_{m}-y_{m}\right)\right. \\
& +(t-s)^{\frac{\alpha-1}{\alpha}} s^{\frac{\beta-\alpha}{\alpha}} \rho_{0}^{0}\left(t, x_{m}-y_{m}\right)+(t-s)^{-\frac{1}{\alpha}} \rho_{0}^{\beta}\left(t, x_{m}-y_{m}\right) \\
& \left.+(t-s)^{-\frac{1}{\alpha}} s^{\frac{\beta}{\alpha}} \rho_{0}^{0}\left(t, x_{m}-y_{m}\right)\right] d s . \\
& \leq c \sum_{m=1}^{d}\left[\prod_{\substack{i=1 \\
i \neq m}}^{d} \rho_{\alpha}^{0}\left(t, x_{i}-y_{i}\right)\right]\left[\rho_{\alpha+\beta-1}^{0}\left(t, x_{m}-y_{m}\right)+\rho_{\alpha-1}^{\beta}\left(t, x_{m}-y_{m}\right)\right],
\end{aligned}
$$

which implies (3.32).

Proposition 3.15. For any $\alpha \in(1,2), t \in(0, T]$ and $x, y \in \mathbb{R}^{d}$ we have

$$
\left|\nabla_{x} p^{A}(t, x, y)\right| \leq c t^{-\frac{1}{\alpha}} \prod_{i=1}^{d} g_{t}\left(x_{i}-y_{i}\right) .
$$

Proof: The assertion follows from formula (3.30) and Lemmas 3.11, 3.14.

\section{Feller semigroup}

For any bounded Borel $f: \mathbb{R}^{d} \rightarrow \mathbb{R}, t \in(0, \infty)$ and $x \in \mathbb{R}^{d}$ we define

$$
P_{t}^{A} f(x)=\int_{\mathbb{R}^{d}} p^{A}(t, x, y) f(y) d y .
$$

The main aim of this section is to show that $\left\{P_{t}^{A}\right\}$ is a Feller semigroup. 
For any $\varepsilon \geq 0$ and $x \in \mathbb{R}^{d}$ we put

$$
\begin{aligned}
& \mathcal{L}_{\varepsilon} f(x)=\frac{\mathcal{A}_{\alpha}}{2} \sum_{i=1}^{d} \int_{\left\{z_{i}:\left|z_{i}\right|>\varepsilon\right\}} \delta_{f}\left(x, e_{i} z_{i}\right) \sigma_{i}(x) \frac{d z_{i}}{\left|z_{i}\right|^{1+\alpha}}, \\
& \mathcal{L}_{\varepsilon}^{y} f(x)=\frac{\mathcal{A}_{\alpha}}{2} \sum_{i=1}^{d} \int_{\left\{z_{i}:\left|z_{i}\right|>\varepsilon\right\}} \delta_{f}\left(x, e_{i} z_{i}\right) \sigma_{i}(y) \frac{d z_{i}}{\left|z_{i}\right|^{1+\alpha}} .
\end{aligned}
$$

Using Chen and Zhang (2016, (3.13)) and the same arguments as in the proof of Lemma 3.12 we obtain

Lemma 4.1. For any $\varepsilon>0, x, y \in \mathbb{R}^{d}$ and $t \in(0, T]$ we have

$$
\left|\int_{\mathbb{R}^{d}} \mathcal{L}_{\varepsilon}^{x} p_{y}(t, \cdot)(x-y) d y\right| \leq c t^{\frac{\beta-\alpha}{\alpha}} .
$$

Lemma 4.2. For any $x, y \in \mathbb{R}^{d}$ and $t>0 \mathcal{L}^{x} \varphi_{y}(t, x)$ is well defined and we have

$$
\mathcal{L}^{x} \varphi_{y}(t, x)=\int_{0}^{t} \int_{\mathbb{R}^{d}} \mathcal{L}^{x} p_{z}(t-s, \cdot)(x-z) q(s, z, y) d z d s .
$$

Fix $\gamma \in(0, \beta)$. There exists $c$ such that for any $\varepsilon>0, t \in(0, T]$ and $x, y \in \mathbb{R}^{d}$ we have

$$
\left|\mathcal{L}_{\varepsilon} p^{A}(t, \cdot, y)(x)\right| \leq c t^{\frac{-\alpha+\gamma-\beta}{\alpha}} \prod_{i=1}^{d} \rho_{\alpha}^{0}\left(t, x_{i}-y_{i}\right) .
$$

Moreover, $t \rightarrow \mathcal{L}^{x} \varphi_{y}(t, x)$ is continuous on $(0, T)$ for any $x, y \in \mathbb{R}^{d}$.

Proof: Let $\varepsilon>0$. We have

$$
\mathcal{L}_{\varepsilon} \varphi_{y}(t, x)=\int_{0}^{t} \int_{\mathbb{R}^{d}} \mathcal{L}_{\varepsilon}^{x} p_{z}(t-s, \cdot)(x-z) q(s, z, y) d z d s .
$$

By Lemma 3.1 and Theorem 3.2 one easily gets

$$
\lim _{\varepsilon \rightarrow 0^{+}} \int_{\mathbb{R}^{d}} \mathcal{L}_{\varepsilon}^{x} p_{z}(t-s, \cdot)(x-z) q(s, z, y) d z=\int_{\mathbb{R}^{d}} \mathcal{L}^{x} p_{z}(t-s, \cdot)(x-z) q(s, z, y) d z .
$$

The most difficult part of the proof is to justify

$$
\begin{aligned}
& \lim _{\varepsilon \rightarrow 0^{+}} \int_{0}^{t} \int_{\mathbb{R}^{d}} \mathcal{L}_{\varepsilon}^{x} p_{z}(t-s, \cdot)(x-z) q(s, z, y) d z d s \\
& =\int_{0}^{t} \lim _{\varepsilon \rightarrow 0^{+}} \int_{\mathbb{R}^{d}} \mathcal{L}_{\varepsilon}^{x} p_{z}(t-s, \cdot)(x-z) q(s, z, y) d z d s .
\end{aligned}
$$

We have

$$
\begin{aligned}
\mathcal{L}_{\varepsilon} \varphi_{y}(t, x) & =\int_{0}^{t / 2} \int_{\mathbb{R}^{d}} \mathcal{L}_{\varepsilon}^{x} p_{z}(t-s, \cdot)(x-z) q(s, z, y) d z d s \\
& +\int_{t / 2}^{t} \int_{\mathbb{R}^{d}} \mathcal{L}_{\varepsilon}^{x} p_{z}(t-s, \cdot)(x-z) d z q(s, x, y) d s \\
& +\int_{t / 2}^{t} \int_{\mathbb{R}^{d}} \mathcal{L}_{\varepsilon}^{x} p_{z}(t-s, \cdot)(x-z)(q(s, z, y)-q(s, x, y)) d z d s \\
& =D(t, x, y)+E(t, x, y)+F(t, x, y) .
\end{aligned}
$$


For $s \in(0, t / 2)$ by Theorem 3.2, Lemma 3.1 and (2.4) we obtain

$$
\begin{aligned}
& \int_{\mathbb{R}^{d}}\left|\mathcal{L}_{\varepsilon}^{x} p_{z}(t-s, \cdot)(x-z) q(s, z, y)\right| d z \\
& \leq c \sum_{m=1}^{d}\left[\prod_{\substack{i=1 \\
i \neq m}}^{d} \rho_{\alpha}^{0}\left(t, x_{i}-y_{i}\right)\right] \\
& \times \int_{\mathbb{R}} \rho_{0}^{0}\left(t-s, x_{m}-z_{m}\right)\left[\rho_{0}^{\beta}\left(s, z_{m}-y_{m}\right)+\rho_{\beta}^{0}\left(s, z_{m}-y_{m}\right)\right] d z \\
& \leq c \sum_{m=1}^{d}\left[\prod_{\substack{i=1 \\
i \neq m}}^{d} \rho_{\alpha}^{0}\left(t, x_{i}-y_{i}\right)\right] \\
& \times\left[(t-s)^{\frac{\beta-\alpha}{\alpha}} \rho_{0}^{0}\left(t, x_{m}-y_{m}\right)+s^{\frac{\beta-\alpha}{\alpha}} \rho_{0}^{0}\left(t, x_{m}-y_{m}\right)+(t-s)^{-1} \rho_{0}^{\beta}\left(t, x_{m}-y_{m}\right)\right] .
\end{aligned}
$$

It follows that

$$
\begin{aligned}
& \lim _{\varepsilon \rightarrow 0^{+}} \int_{0}^{t / 2} \int_{\mathbb{R}^{d}} \mathcal{L}_{\varepsilon}^{x} p_{z}(t-s, \cdot)(x-z) q(s, z, y) d z d s \\
& =\int_{0}^{t / 2} \lim _{\varepsilon \rightarrow 0^{+}} \int_{\mathbb{R}^{d}} \mathcal{L}_{\varepsilon}^{x} p_{z}(t-s, \cdot)(x-z) q(s, z, y) d z d s .
\end{aligned}
$$

and

$$
D(t, x, y) \leq c t^{-1} \prod_{i=1}^{d} \rho_{\alpha}^{0}\left(t, x_{i}-y_{i}\right)
$$

For $s \in(t / 2, t)$ by Theorem 3.2 and Lemma 4.1 we obtain

$$
\left|\int_{\mathbb{R}^{d}} \mathcal{L}_{\varepsilon}^{x} p_{z}(t-s, \cdot)(x-z) d z q(s, x, y)\right| \leq c(t-s)^{\frac{\beta-\alpha}{\alpha}} t^{-1} \prod_{i=1}^{d} \rho_{\alpha}^{0}\left(t, x_{i}-y_{i}\right) .
$$

It follows that

$$
\begin{aligned}
& \lim _{\varepsilon \rightarrow 0^{+}} \int_{t / 2}^{t} \int_{\mathbb{R}^{d}} \mathcal{L}_{\varepsilon}^{x} p_{z}(t-s, \cdot)(x-z) d z q(s, x, y) d s \\
& =\int_{t / 2}^{t} \lim _{\varepsilon \rightarrow 0^{+}} \int_{\mathbb{R}^{d}} \mathcal{L}_{\varepsilon}^{x} p_{z}(t-s, \cdot)(x-z) d z q(s, x, y) d s .
\end{aligned}
$$

and

$$
E(t, x, y) \leq c t^{-1} \prod_{i=1}^{d} \rho_{\alpha}^{0}\left(t, x_{i}-y_{i}\right)
$$

Now, we need to obtain some estimates which will be crucial in studying the most difficult term $F(t, x, y)$. By Lemma 3.1 and Theorem 3.9 there exists $c$ (not depending on $\varepsilon$ ) such that for any $t \in(0, T]$ and $x, y \in \mathbb{R}^{d}$ we have

$$
\int_{\mathbb{R}^{d}} \sum_{i=1}^{d}\left[\int_{\left|w_{i}\right|>\varepsilon}\left|\delta_{p_{z}}\left(t-s, x-z, w_{i} e_{i}\right)\right|\left|w_{i}\right|^{-1-\alpha} d w_{i}|q(s, z, y)-q(s, x, y)|\right] d z
$$




$$
\begin{aligned}
& \leq c(t-s)^{-1} \int_{\mathbb{R}^{d}}\left(|x-z|^{\beta-\gamma} \wedge 1\right)\left[\prod_{i=1}^{d} \rho_{\alpha}^{0}\left(t-s, x_{i}-z_{i}\right)\right] d z \sum_{m=1}^{d}\left[\prod_{\substack{i=1 \\
i \neq m}}^{d} \rho_{\alpha}^{0}\left(s, x_{i}-y_{i}\right)\right] \\
& \times\left[\rho_{\gamma-\beta}^{\beta}\left(s, x_{m}-y_{m}\right)+\rho_{\gamma}^{0}\left(s, x_{m}-y_{m}\right)\right] \\
& +c(t-s)^{-1} \int_{\mathbb{R}^{d}}\left(|x-z|^{\beta-\gamma} \wedge 1\right)\left[\prod_{i=1}^{d} \rho_{\alpha}^{0}\left(t-s, x_{i}-z_{i}\right)\right] \sum_{m=1}^{d}\left[\prod_{\substack{i=1 \\
i \neq m}}^{d} \rho_{\alpha}^{0}\left(s, z_{i}-y_{i}\right)\right] \\
& \times\left[\rho_{\gamma-\beta}^{\beta}\left(s, z_{m}-y_{m}\right)+\rho_{\gamma}^{0}\left(s, z_{m}-y_{m}\right)\right] d z \\
& =B_{1}(s, t, x, y)+B_{2}(s, t, x, y) .
\end{aligned}
$$

Clearly $\left(|x-z|^{\beta-\gamma} \wedge 1\right) \leq \sum_{k=1}^{d}\left(\left|x_{k}-z_{k}\right|^{\beta-\gamma} \wedge 1\right)$. It follows that

$$
\begin{aligned}
& (t-s)^{-1} \int_{\mathbb{R}^{d}}\left(|x-z|^{\beta-\gamma} \wedge 1\right)\left[\prod_{i=1}^{d} \rho_{\alpha}^{0}\left(t-s, x_{i}-z_{i}\right)\right] d z \\
\leq & c \sum_{k=1}^{d} \int_{\mathbb{R}} \rho_{0}^{\beta-\gamma}\left(t-s, x_{k}-z_{k}\right) d z_{k} \\
\leq & c(t-s)^{\frac{\beta-\gamma-\alpha}{\alpha}} .
\end{aligned}
$$

Hence

$$
\begin{aligned}
B_{1}(s, t, x, y) & \leq c(t-s)^{\frac{\beta-\gamma-\alpha}{\alpha}} \sum_{m=1}^{d}\left[\prod_{\substack{i=1 \\
i \neq m}}^{d} \rho_{\alpha}^{0}\left(s, x_{i}-y_{i}\right)\right] \\
& \times\left[\rho_{\gamma-\beta}^{\beta}\left(s, x_{m}-y_{m}\right)+\rho_{\gamma}^{0}\left(s, x_{m}-y_{m}\right)\right] .
\end{aligned}
$$

We also have

$$
\begin{aligned}
B_{2}(s, t, x, y) & \leq c \sum_{m=1}^{d} \int_{\mathbb{R}^{d}}\left[\prod_{\substack{i=1 \\
i \neq m}}^{d} \rho_{\alpha}^{0}\left(t-s, x_{i}-z_{i}\right) \rho_{\alpha}^{0}\left(s, z_{i}-y_{i}\right)\right] \\
& \times \rho_{0}^{\beta-\gamma}\left(t-s, x_{m}-z_{m}\right)\left[\rho_{\gamma-\beta}^{\beta}\left(s, z_{m}-y_{m}\right)+\rho_{\gamma}^{0}\left(s, z_{m}-y_{m}\right)\right] d z \\
& +c \sum_{m=1}^{d} \sum_{\substack{k=1 \\
k \neq m}}^{d} \int_{\mathbb{R}^{d}}\left[\prod_{\substack{i=1 \\
i \neq m, k}}^{d} \rho_{\alpha}^{0}\left(t-s, x_{i}-z_{i}\right) \rho_{\alpha}^{0}\left(s, z_{i}-y_{i}\right)\right] \\
& \times \rho_{\alpha}^{0}\left(t-s, x_{m}-z_{m}\right)\left[\rho_{\gamma-\beta}^{\beta}\left(s, z_{m}-y_{m}\right)+\rho_{\gamma}^{0}\left(s, z_{m}-y_{m}\right)\right] \\
& \times \rho_{0}^{\beta-\gamma}\left(t-s, x_{k}-z_{k}\right) \rho_{\alpha}^{0}\left(s, z_{k}-y_{k}\right) d z \\
& =B_{3}(s, t, x, y)+B_{4}(s, t, x, y) .
\end{aligned}
$$

By (2.4), we have

$$
\begin{aligned}
& B_{3}(s, t, x, y) \\
& \leq c \sum_{m=1}^{d}\left[\prod_{\substack{i=1 \\
i \neq m}}^{d} \rho_{\alpha}^{0}\left(t, x_{i}-y_{i}\right)\right]\left[(t-s)^{\frac{2 \beta-\gamma-\alpha}{\alpha}} s^{\frac{\gamma-\beta}{\alpha}} \rho_{0}^{0}\left(t, x_{m}-y_{m}\right)+s^{\frac{\beta-\alpha}{\alpha}} \rho_{0}^{0}\left(t, x_{m}-y_{m}\right)\right.
\end{aligned}
$$




$$
\begin{aligned}
& +(t-s)^{\frac{\beta-\gamma-\alpha}{\alpha}} s^{\frac{\gamma-\beta}{\alpha}} \rho_{0}^{\beta}\left(t, x_{m}-y_{m}\right)+s^{\frac{\gamma-\alpha}{\alpha}} \rho_{0}^{\beta-\gamma}\left(t, x_{m}-y_{m}\right) \\
& \left.+(t-s)^{\frac{\beta-\gamma-\alpha}{\alpha}} s^{\frac{\gamma}{\alpha}} \rho_{0}^{0}\left(t, x_{m}-y_{m}\right)\right] .
\end{aligned}
$$

By (2.4), we also have

$$
\begin{aligned}
& B_{4}(s, t, x, y) \\
& \leq c \sum_{m=1}^{d} \sum_{\substack{k=1 \\
k \neq m}}^{d}\left[\prod_{\substack{i=1 \\
i \neq m, k}}^{d} \rho_{\alpha}^{0}\left(t, x_{i}-y_{i}\right)\right] \\
& \times\left[\left[(t-s)^{\frac{\beta}{\alpha}} s^{\frac{\gamma-\beta}{\alpha}}+(t-s)^{\frac{\alpha}{\alpha}} s^{\frac{\gamma-\alpha}{\alpha}}+s^{\frac{\gamma}{\alpha}}\right] \rho_{0}^{0}\left(t, x_{m}-y_{m}\right)+s^{\frac{\gamma-\beta}{\alpha}} \rho_{0}^{\beta}\left(t, x_{m}-y_{m}\right)\right] \\
& \times\left[\left[(t-s)^{\frac{\beta-\gamma-\alpha}{\alpha}} s^{\frac{\alpha}{\alpha}}+s^{\frac{\beta-\gamma}{\alpha}}\right] \rho_{0}^{0}\left(t, x_{k}-y_{k}\right)+\rho_{0}^{\beta-\gamma}\left(t, x_{k}-y_{k}\right)\right]
\end{aligned}
$$

By (4.9-4.11) and (2.3), we get

$$
\begin{aligned}
& \lim _{\varepsilon \rightarrow 0^{+}} \int_{t / 2}^{t} \int_{\mathbb{R}^{d}} \mathcal{L}_{\varepsilon}^{x} p_{z}(t-s, \cdot)(x-z)(q(s, z, y)-q(s, x, y)) d z d s \\
& =\int_{t / 2}^{t} \lim _{\varepsilon \rightarrow 0^{+}} \int_{\mathbb{R}^{d}} \mathcal{L}_{\varepsilon}^{x} p_{z}(t-s, \cdot)(x-z)(q(s, z, y)-q(s, x, y)) d z d s .
\end{aligned}
$$

and

$$
F(t, x, y) \leq c t^{\frac{-\alpha+\gamma-\beta}{\alpha}} \prod_{i=1}^{d} \rho_{\alpha}^{0}\left(t, x_{i}-y_{i}\right) .
$$

By (4.5), (4.7), (4.12) we get (4.4). We also get continuity $t \rightarrow \mathcal{L}^{x} \varphi_{y}(t, x)$. By (4.3) and (4.4) we obtain (4.1). Using (4.6), (4.8), (4.13), Lemma 3.1 and formula (3.30) we get (4.2).

The next result is an analogue of Chen and Zhang (2016, Theorem 4.1). Its proof is almost the same as the proof of Chen and Zhang (2016, Theorem 4.1) and is omitted.

Proposition 4.3. Let $u(t, x) \in C_{b}\left([0, T] \times \mathbb{R}^{d}\right)$ with

$$
\lim _{t \rightarrow 0^{+}} \sup _{x \in \mathbb{R}^{d}}|u(t, x)-u(0, x)|=0 .
$$

Assume that

$$
t \rightarrow \mathcal{L} u(t, x) \quad \text { is continuous on } \quad(0, T] \text { for each } x \in \mathbb{R}^{d}
$$

and for any $\varepsilon \in(0,1)$ and some $\gamma \in((\alpha-1) \vee 0,1)$

$$
\sup _{t \in(\varepsilon, T)}\left|u(t, x)-u\left(t, x^{\prime}\right)\right| \leq K_{\varepsilon}\left|x-x^{\prime}\right|^{\gamma}, \quad x, x^{\prime} \in \mathbb{R}^{d} .
$$

If $u$ satisfies

$$
\frac{\partial}{\partial t} u(t, x)=\mathcal{L} u(t, x), \quad t \in(0, T], x \in \mathbb{R}^{d},
$$

then

$$
\sup _{t \in(0, T)} \sup _{x \in \mathbb{R}^{d}} u(t, x) \leq \sup _{x \in \mathbb{R}^{d}} u(0, x) .
$$


Lemma 4.4. Let $x, y \in \mathbb{R}^{d}$. Then the mapping $t \rightarrow \varphi_{y}(t, x)$ is absolutely continuous on $(0, T]$. For any $t \in(0, T)$ we have

$$
\frac{\partial \varphi_{y}}{\partial t}(t, x)=q(t, x, y)+\int_{0}^{t} \int_{\mathbb{R}^{d}} \mathcal{L}^{z} p_{z}(t-s, \cdot)(x-z) q(s, z, y) d z d s .
$$

Proof: Let $h>0$ be such that $t+h<T$. We have

$$
\begin{aligned}
& \frac{\varphi_{y}(t+h, x)-\varphi_{y}(t, x)}{h} \\
& =\frac{1}{h} \int_{0}^{t+h} \int_{\mathbb{R}^{d}} p_{z}(t+h-s, x-z) q(s, z, y) d z d s \\
& -\frac{1}{h} \int_{0}^{t} \int_{\mathbb{R}^{d}} p_{z}(t-s, x-z) q(s, z, y) d z d s \\
& =\frac{1}{h} \int_{0}^{t+h} \int_{\mathbb{R}^{d}} p_{z}(t+h-s, x-z) q(s, z, y) d z d s \\
& -\frac{1}{h} \int_{0}^{t} \int_{\mathbb{R}^{d}} p_{z}(t+h-s, x-z) q(s, z, y) d z d s \\
& +\frac{1}{h} \int_{0}^{t} \int_{\mathbb{R}^{d}} p_{z}(t+h-s, x-z) q(s, z, y) d z d s \\
& -\frac{1}{h} \int_{0}^{t} \int_{\mathbb{R}^{d}} p_{z}(t-s, x-z) q(s, z, y) d z d s \\
& =\frac{1}{h} \int_{t}^{t+h} \int_{\mathbb{R}^{d}} p_{z}(t+h-s, x-z) q(s, z, y) d z d s \\
& +\int_{0}^{t} \int_{\mathbb{R}^{d}} \frac{p_{z}(t+h-s, x-z)-p_{z}(t-s, x-z)}{h} q(s, z, y) d z d s \\
& =\mathrm{I}+\mathrm{II}
\end{aligned}
$$

After change of variables $t+h-s=u$ we have

$$
\begin{aligned}
\mathrm{I} & =\frac{1}{h} \int_{0}^{h} \int_{\mathbb{R}^{d}} p_{z}(u, x-z) q(t+h-u, z, y) d z d u \\
& =\frac{1}{h} \int_{0}^{h} \int_{\mathbb{R}^{d}}\left(p_{z}(u, x-z)-p_{x}(u, x-z)\right) q(t+h-u, z, y) d z d u \\
& +\frac{1}{h} \int_{0}^{h} \int_{\mathbb{R}^{d}} p_{x}(u, x-z) q(t+h-u, z, y) d z d u \\
& =\mathrm{I}_{1}+\mathrm{I}_{2} .
\end{aligned}
$$

By Theorem $3.2, \sup _{u \leq h, z \in \mathbb{R}^{d}} q(t+h-u, z, y) \leq M<\infty$. Moreover, from Corollary 3.5,

$$
\left|p_{z}(u, x-z)-p_{x}(u, x-z)\right| \leq c p_{x}(u, x-z)\left(|x-z|^{\beta} \wedge 1\right) .
$$

Hence,

$$
\limsup _{h \rightarrow 0^{+}}\left|\mathrm{I}_{1}\right| \leq c M \limsup _{h \rightarrow 0^{+}} \frac{1}{h} \int_{0}^{h} \int_{\mathbb{R}^{d}} p_{x}(u, x-z)\left(|x-z|^{\beta} \wedge 1\right) d z d u=0 .
$$

Next, by Theorem 3.2, the function $q(s, z, y)$ is continuous and bounded on $[t, T] \times$ $\mathbb{R}^{d}$, as a function of $s$ and $z$. Since the measures $\mu_{u}(d z)=p_{x}(u, x-z) d z$ converge 
weakly to $\delta_{x}$ as $u \rightarrow 0^{+}$, we obtain

$$
\lim _{h \rightarrow 0^{+}} \mathrm{I}_{2}=q(t, x, y) .
$$

We have

$$
\begin{aligned}
\mathrm{II} & =\int_{0}^{t / 2} \int_{\mathbb{R}^{d}} \frac{p_{z}(t+h-s, x-z)-p_{z}(t-s, x-z)}{h} q(s, z, y) d z d s \\
& +\int_{t / 2}^{t} \int_{\mathbb{R}^{d}} \frac{p_{z}(t+h-s, x-z)-p_{z}(t-s, x-z)}{h} q(s, z, y) d z d s \\
& =\mathrm{III}+\mathrm{IV} .
\end{aligned}
$$

By estimates of $\frac{\partial}{\partial t} p_{z}(t-s, x-z)$ following from (3.3) and Theorem 3.2 we get

$$
\lim _{h \rightarrow 0^{+}} \mathrm{III}=\int_{0}^{t / 2} \int_{\mathbb{R}^{d}} \frac{\partial}{\partial t} p_{z}(t-s, x-z) q(s, z, y) d z d s .
$$

We have

$$
\begin{aligned}
\mathrm{IV} & =\int_{t / 2}^{t} \int_{\mathbb{R}^{d}} \frac{p_{z}(t+h-s, x-z)-p_{z}(t-s, x-z)}{h}(q(s, z, y)-q(s, x, y)) d z d s \\
& +\int_{t / 2}^{t} \int_{\mathbb{R}^{d}} \frac{p_{z}(t+h-s, x-z)-p_{z}(t-s, x-z)}{h} d z q(s, x, y) d s \\
& =\mathrm{V}+\mathrm{VI} .
\end{aligned}
$$

Note that for $h>0, s \in(t / 2, t), \gamma \in(0, \beta), x, y, z \in \mathbb{R}^{d}$, by Theorem 3.9 and the estimates of $\frac{\partial}{\partial t} p_{z}(t-s, x-z)$, we obtain

$$
\begin{aligned}
& \left|\frac{p_{z}(t+h-s, x-z)-p_{z}(t-s, x-z)}{h}\right||(q(s, z, y)-q(s, x, y))| \\
\leq & B\left(|x-z|^{\beta-\gamma} \wedge 1\right)\left|\frac{\partial}{\partial t} p_{z}(t+\theta h-s, x-z)\right| \\
\leq & c B\left(|x-z|^{\beta-\gamma} \wedge 1\right)(t-s)^{-1} \prod_{i=1}^{d} \rho_{\alpha}^{0}\left(t-s, x_{i}-z_{i}\right),
\end{aligned}
$$

where $B=B\left(t, \alpha, d, b_{1}, b_{2}, b_{3}, \beta, \gamma\right) \in(0, \infty)$ and $\theta=\theta\left(s, t, h, \alpha, d, b_{1}, b_{2}, b_{3}, \beta\right.$, $\gamma, x, z) \in(0,1)$. We also have

$$
\begin{aligned}
& \int_{\mathbb{R}^{d}}\left(|x-z|^{\beta-\gamma} \wedge 1\right)(t-s)^{-1} \prod_{i=1}^{d} \rho_{\alpha}^{0}\left(t-s, x_{i}-z_{i}\right) d z \\
\leq & c \sum_{i=1}^{d} \int_{\mathbb{R}} \rho_{0}^{\beta-\gamma}\left(t-s, x_{i}-z_{i}\right) d z_{i} \\
\leq & c(t-s)^{\frac{\beta-\gamma-\alpha}{\alpha}} .
\end{aligned}
$$

It follows that

$$
\lim _{h \rightarrow 0^{+}} \mathrm{V}=\int_{t / 2}^{t} \int_{\mathbb{R}^{d}} \frac{\partial}{\partial t} p_{z}(t-s, x-z)(q(s, z, y)-q(s, x, y)) d z d s .
$$

Note that for $h>0, s \in(t / 2, t), x, z \in \mathbb{R}^{d}$ we have

$$
\frac{1}{h}\left(\int_{\mathbb{R}^{d}} p_{z}(t+h-s, x-z) d z-\int_{\mathbb{R}^{d}} p_{z}(t-s, x-z) d z\right)
$$




$$
\begin{aligned}
& =\frac{\partial}{\partial t} \int_{\mathbb{R}^{d}} p_{z}(t+\theta h-s, x-z) d z \\
& =\int_{\mathbb{R}^{d}} \frac{\partial}{\partial t} p_{z}(t+\theta h-s, x-z) d z,
\end{aligned}
$$

where $\theta=\theta\left(s, t, h, \alpha, d, b_{1}, b_{2}, b_{3}, \beta, \gamma, x\right) \in(0,1)$.

Using this, (2.6) and the definition of $q_{0}(t, x, y)$ we get

$$
\begin{aligned}
& \int_{\mathbb{R}^{d}} \frac{p_{z}(t+h-s, x-z)-p_{z}(t-s, x-z)}{h} d z \\
= & \int_{\mathbb{R}^{d}} \mathcal{L}^{z} p_{z}(t+\theta h-s, \cdot)(x-z) d z \\
= & -\int_{\mathbb{R}^{d}} q_{0}(t+\theta h-s, x, z) d z+\int_{\mathbb{R}^{d}} \mathcal{L}^{x} p_{z}(t+\theta h-s, \cdot)(x-z) d z .
\end{aligned}
$$

By (3.5) and (2.3), we have

$$
\left|\int_{\mathbb{R}^{d}} q_{0}(t+\theta h-s, x, z) d z\right| \leq c(t-s)^{\frac{\beta-\alpha}{\alpha}} .
$$

By Lemma 4.1 we get

$$
\left|\int_{\mathbb{R}^{d}} \mathcal{L}^{x} p_{z}(t+\theta h-s, \cdot)(x-z) d z\right| \leq c(t-s)^{\frac{\beta-\alpha}{\alpha}} .
$$

It follows that

$$
\lim _{h \rightarrow 0^{+}} \mathrm{VI}=\int_{t / 2}^{t} \int_{\mathbb{R}^{d}} \frac{\partial}{\partial t} p_{z}(t-s, x-z) d z q(s, x, y) d s .
$$

By (4.20-4.24) we obtain

$$
\lim _{h \rightarrow 0^{+}} \frac{\varphi_{y}(t+h, x)-\varphi_{y}(t, x)}{h}=q(t, x, y)+\int_{0}^{t} \int_{\mathbb{R}^{d}} \mathcal{L}^{z} p_{z}(t-s, \cdot)(x-z) q(s, z, y) d z d s .
$$

The proof of the analogous result for $\lim _{h \rightarrow 0^{-}}$is very similar and it is omitted.

Proposition 4.5. For all $t \in(0, \infty)$ and $x, y \in \mathbb{R}^{d}$ we have

$$
\frac{\partial}{\partial t} p^{A}(t, x, y)=\mathcal{L} p^{A}(t, \cdot, y)(x)
$$

Proof: By the definition of $q(t, x, y)$ we obtain

$$
q(t, x, y)=q_{0}(t, x, y)+\int_{0}^{t} \int_{\mathbb{R}^{d}} q_{0}(t-s, x, z) q(s, z, y) d z d s .
$$

Using (3.30), (2.6), Lemma 4.4 and the definition of $q_{0}(t, x, y)$ we obtain

$$
\begin{aligned}
& \frac{\partial p^{A}}{\partial t}(t, x, y)=\frac{\partial p_{y}}{\partial t}(t, x-y)+\frac{\partial \varphi_{y}}{\partial t}(t, x) \\
& =\mathcal{L}^{y} p_{y}(t, x-y)+q(t, x, y)+\int_{0}^{t} \int_{\mathbb{R}^{d}} \mathcal{L}^{z} p_{z}(t-s, \cdot)(x-z) q(s, z, y) d z d s \\
& =\mathcal{L}^{x} p_{y}(t, x-y)-q_{0}(t, x, y)+q(t, x, y)+\int_{0}^{t} \int_{\mathbb{R}^{d}} \mathcal{L}^{z} p_{z}(t-s, \cdot)(x-z) q(s, z, y) d z d s .
\end{aligned}
$$

By (4.25) this is equal to

$$
\mathcal{L}^{x} p_{y}(t, x-y)+\int_{0}^{t} \int_{\mathbb{R}^{d}}\left(q_{0}(t-s, x, z)+\mathcal{L}^{z} p_{z}(t-s, \cdot)(x-z)\right) q(s, z, y) d z d s
$$




$$
=\mathcal{L}^{x} p_{y}(t, x-y)+\int_{0}^{t} \int_{\mathbb{R}^{d}} \mathcal{L}^{x} p_{z}(t-s, \cdot)(x-z) q(s, z, y) d z d s .
$$

By (4.1) and (3.30) this is equal to $\mathcal{L}^{x} p^{A}(t, \cdot, y)(x)$, which completes the proof.

Lemma 4.6. For any bounded Borel $f: \mathbb{R}^{d} \rightarrow \mathbb{R}, t \in(0, \infty)$ and $x \in \mathbb{R}^{d}$ we have

$$
\mathcal{L}\left(P_{t}^{A} f\right)(x)=\frac{\partial}{\partial t} P_{t}^{A} f(x) .
$$

Proof: We have

$$
\mathcal{L}\left(P_{t}^{A} f\right)(x)=\lim _{\varepsilon \rightarrow 0^{+}} \mathcal{L}_{\varepsilon}\left(P_{t}^{A} f\right)(x)=\lim _{\varepsilon \rightarrow 0^{+}} \int_{\mathbb{R}^{d}} \mathcal{L}_{\varepsilon} p^{A}(t, \cdot, y)(x) f(y) d y .
$$

By Lemma 4.2 this is equal to

$$
\int_{\mathbb{R}^{d}} \lim _{\varepsilon \rightarrow 0^{+}} \mathcal{L}_{\varepsilon} p^{A}(t, \cdot, y)(x) f(y) d y=\int_{\mathbb{R}^{d}} \mathcal{L} p^{A}(t, \cdot, y)(x) f(y) d y .
$$

By Proposition 4.5 this is equal to

$$
\int_{\mathbb{R}^{d}} \frac{\partial}{\partial t} p^{A}(t, x, y) f(y) d y=\frac{\partial}{\partial t} \int_{\mathbb{R}^{d}} p^{A}(t, x, y) f(y) d y
$$

Proposition 4.7. For $t \in(0, T]$ and $x, y \in \mathbb{R}^{d}$ we have

$$
p^{A}(t, x, y) \leq c \prod_{i=1}^{d} g_{t}\left(x_{i}-y_{i}\right) .
$$

Proof: By Theorem 3.2, estimates of $p_{z}$ and (2.5) we obtain

$$
\begin{aligned}
& \left|\varphi_{y}(t, x)\right|=\left|\int_{0}^{t} \int_{\mathbb{R}^{d}} p_{z}(t-s, x-z) q(s, z, y) d z d s\right| \\
& \leq c \int_{0}^{t} \int_{\mathbb{R}^{d}} \prod_{i=1}^{d} \rho_{\alpha}^{0}\left(t-s, x_{i}-z_{i}\right) \\
& \times \sum_{m=1}^{d}\left[\prod_{\substack{i=1 \\
i \neq m}}^{d} \rho_{\alpha}^{0}\left(s, z_{i}-y_{i}\right)\right]\left[\rho_{0}^{\beta}\left(s, z_{m}-y_{m}\right)+\rho_{\beta}^{0}\left(s, z_{m}-y_{m}\right)\right] d z d s \\
& \leq c \sum_{m=1}^{d}\left[\prod_{\substack{i=1 \\
i \neq m}}^{d} \rho_{\alpha}^{0}\left(t, x_{i}-y_{i}\right)\right] \int_{0}^{t} \int_{\mathbb{R}} \rho_{\alpha}^{0}\left(t-s, x_{m}-z_{m}\right) \\
& \times\left[\rho_{0}^{\beta}\left(s, z_{m}-y_{m}\right)+\rho_{\beta}^{0}\left(s, z_{m}-y_{m}\right)\right] d z d s \\
& \leq c \sum_{m=1}^{d}\left[\prod_{\substack{i=1 \\
i \neq m}}^{d} \rho_{\alpha}^{0}\left(t, x_{i}-y_{i}\right)\right]\left[\rho_{\alpha+\beta}^{0}\left(t, x_{m}-y_{m}\right)+\rho_{\alpha}^{\beta}\left(t, x_{m}-y_{m}\right)\right] \\
& \leq c\left[\prod_{i=1}^{d} \rho_{\alpha}^{0}\left(t, x_{i}-y_{i}\right)\right]\left[t^{\beta / \alpha}+\sum_{m=1}^{d}\left(\left|x_{m}-y_{m}\right|^{\beta} \wedge 1\right)\right] .
\end{aligned}
$$

Now the conlusion follows from (3.2) and estimates of $p_{y}$. 
The following result shows that $\left\{P_{t}^{A}\right\}$ is a Feller semigroup.

Theorem 4.8. We have:

(i) $P_{t}^{A}: C_{0}\left(\mathbb{R}^{d}\right) \rightarrow C_{0}\left(\mathbb{R}^{d}\right)$ for any $t \in(0, \infty)$,

(ii) $\lim _{t \rightarrow 0^{+}}\left\|P_{t}^{A} f-f\right\|_{\infty}=0$ for any $f \in C_{0}\left(\mathbb{R}^{d}\right)$.

(iii) $p^{A}(t, x, y) \geq 0$ for any $(t, x, y) \in(0, \infty) \times \mathbb{R}^{d} \times \mathbb{R}^{d}$,

(iv) $\int_{\mathbb{R}^{d}} p^{A}(t, x, y) d y=1$ for any $(t, x) \in(0, \infty) \times \mathbb{R}^{d}$,

(v) $\int_{\mathbb{R}^{d}} p^{A}(t, x, z) p^{A}(s, z, y) d z=p^{A}(s+t, x, y)$ for any $(s, t, x, y) \in(0, \infty) \times$ $(0, \infty) \times \mathbb{R}^{d} \times \mathbb{R}^{d}$.

Proof: (i) follows by the fact that $x \rightarrow p^{A}(t, x, y)$ is continuous and by Proposition 4.7.

It is shown in the proof of Proposition 4.7 that

$$
\left|\varphi_{y}(t, x)\right| \leq c \sum_{m=1}^{d}\left[\prod_{\substack{i=1 \\ i \neq m}}^{d} \rho_{\alpha}^{0}\left(t, x_{i}-y_{i}\right)\right]\left[\rho_{\alpha+\beta}^{0}\left(t, x_{m}-y_{m}\right)+\rho_{\alpha}^{\beta}\left(t, x_{m}-y_{m}\right)\right] \text {. }
$$

Let $f \in C_{0}\left(\mathbb{R}^{d}\right)$. It follows that $\lim _{t \rightarrow 0^{+}} \sup _{x \in \mathbb{R}^{d}}\left|\int_{\mathbb{R}^{d}} \varphi_{y}(t, x) f(y) d y\right|=0$ for any $f \in C_{0}\left(\mathbb{R}^{d}\right)$. It is clear that $\lim _{t \rightarrow 0^{+}} \sup _{x \in \mathbb{R}^{d}}\left|\int_{\mathbb{R}^{d}} p_{y}(t, x-y) f(y) d y-f(x)\right|=0$ for any $f \in C_{0}\left(\mathbb{R}^{d}\right)$. Hence we obtain (ii).

For any $(t, x) \in(0, T] \times \mathbb{R}^{d}$ put $u(t, x)=P_{t}^{A} f(x), u(0, x)=f(x)$. Note that $u(t, x)$ satisfies the assumptions of Proposition 4.3. Indeed, (ii) gives (4.14). By Lemma 4.2 we get (4.15). By Theorem 1.1 (iv) and Proposition 3.15 we obtain (4.16). Lemma 4.6 gives (4.17). Applying Proposition 4.3 to $f \in C_{c}^{\infty}, f \leq 0$ we obtain (iii). Note that $\tilde{u}(t, x)=-1+P_{t}^{A} 1(x), u(0, x)=0$ also satisfies the assumptions of Proposition 4.3. Using this proposition we get that $P_{t}^{A} 1 \equiv 1$ which implies (iv). Fix $s \in(0, T], f \in C_{c}^{\infty}, f \geq 0$ and denote $u_{1}(t, x)=P_{t+s}^{A} f(x)$, $u_{2}(t, x)=P_{t}^{A} P_{s}^{A} f(x), u_{1}(0, x)=u_{2}(0, x)=P_{s}^{A} f(x), u(t, x)=u_{1}(t, x)-u_{2}(t, x)$. By Proposition 4.3 applied to $u(t, x)$ we get $u_{1} \equiv u_{2}$ which implies (v).

Using similar ideas as in the proof of (4.1) one can easily obtain the following result.

Lemma 4.9. For any $t \in(0, \infty), x \in \mathbb{R}^{d}$ and any bounded, Hölder continuous function $f$ we have

$$
\mathcal{L}\left[\int_{0}^{t} P_{s}^{A} f(\cdot) d s\right](x)=\int_{0}^{t} \mathcal{L} P_{s}^{A} f(x) d s .
$$

Proposition 4.10. For any $t \in(0, \infty), x \in \mathbb{R}^{d}$ and $f \in C_{b}^{2}\left(\mathbb{R}^{d}\right)$ we have

$$
P_{t}^{A} f(x)=f(x)+\int_{0}^{t} P_{s}^{A} \mathcal{L} f(x) d s .
$$

Proof: Put $u(t, x)=f(x)+\int_{0}^{t} P_{s}^{A} \mathcal{L} f(x) d s$. By (4.28) we get

$$
\mathcal{L} u(t, x)=\mathcal{L} f(x)+\int_{0}^{t} \mathcal{L}\left(P_{s}^{A} \mathcal{L} f\right)(x) d s .
$$

By Lemma 4.6 this is equal to

$$
\mathcal{L} f(x)+\int_{0}^{t} \frac{\partial}{\partial s}\left(P_{s}^{A} \mathcal{L} f\right)(x) d s=P_{t}^{A} \mathcal{L} f(x)=\frac{\partial}{\partial t} u(t, x) .
$$


It is easy to check that $u(t, x)$ satisfies (4.14-4.16). Put $\tilde{u}(t, x)=P_{t}^{A} f(x), \tilde{u}(0, x)=$ $f(x)$ and $v(t, x)=u(t, x)-\tilde{u}(t, x)$. By the arguments from the proof of Theorem 4.8 we obtain that $\tilde{u}(t, x)$ satisfies (4.14-4.17). Using Proposition 4.3 for $v(t, x)$ we get $v \equiv 0$ which implies the assertion of the lemma.

The next theorem gives that $\mathcal{L}$ is a generator of the semigroup $\left\{P_{t}^{A}\right\}$.

Theorem 4.11. For any $f \in C_{b}^{2}\left(\mathbb{R}^{d}\right)$ we have

$$
\lim _{t \rightarrow 0^{+}} \frac{P_{t}^{A} f(x)-f(x)}{t}=\mathcal{L} f(x), \quad x \in \mathbb{R}^{d}
$$

and the convergence is uniform.

Proof: By Proposition 4.10 we have

$$
\lim _{t \rightarrow 0^{+}} \frac{P_{t}^{A} f(x)-f(x)}{t}=\lim _{t \rightarrow 0^{+}} \frac{1}{t} \int_{0}^{t} P_{s}^{A} \mathcal{L} f(x) d s .
$$

By Theorem 4.8 (ii) this is equal to $\mathcal{L} f(x)$ and the convergence is uniform.

Remark 4.12. The heat equation in Proposition 4.5 should be understood in the pointwise sense. We do not claim that the function $p^{A}(t, \cdot, y)$ with fixed $t>0$ and $y \in \mathbb{R}^{d}$ is in the domain of the generator of the constructed semigroup. In fact we do not know what is the domain of the generator. This is an interesting question on its own, but we do not address it since our point is to construct the semigroup and to prove its sharp estimates.

We are now in a position to provide the proofs of most of the parts of Theorem 1.1.

Proof of Theorem 1.1 (i), (ii) and the upper bound estimate in (iii): From Theorem 4.8 and Theorem 4.11 we conclude that there is a Feller process $\tilde{X}_{t}$ with the transition kernel $p^{A}(t, x, y)$ and the generator $\mathcal{L}$. Let $\mathbb{P}^{x}, \mathbb{E}^{x}$ be the distribution and expectation for the process starting from $x \in \mathbb{R}^{d}$. First, note that for any function $f \in C_{b}^{2}\left(\mathbb{R}^{d}\right)$, the process

$$
M_{t}^{\tilde{X}, f}=f\left(\tilde{X}_{t}\right)-f\left(\tilde{X}_{0}\right)-\int_{0}^{t} \mathcal{L} f\left(\tilde{X}_{s}\right) d s
$$

is a $\left(\mathbb{P}^{x}, \mathcal{F}_{t}\right)$ martingale, where $\mathcal{F}_{t}$ is a natural filtration. That is $\mathbb{P}^{x}$ solves the martingale problem for $\left(\mathcal{L}, C_{b}^{2}\left(\mathbb{R}^{d}\right)\right)$. On the other hand, according to Bass and Chen (2006, Theorem 6.3), the unique weak solution $X$ to the stochastic equation (1.1) has the law which is the unique solution to the martingale problem for $\left(\mathcal{L}, C_{b}^{2}\left(\mathbb{R}^{d}\right)\right)$. It follows that that $\tilde{X}$ and $X$ have the same law and $p^{A}(t, x, y)$ is the transition kernel of $X$.

The continuity of $p^{A}(t, x, y)$ with respect to all variables follows from Theorem 3.2. Positivity is a consequence of the lower bound in (1.5) which will be proved in the next section. Finally, (ii) follows from Proposition 4.5. The upper bound estimate in (iii) follows from Proposition 4.7.

Proof of Theorem 1.1 (iv): The main tool used in this proof is Theorem 3.2. By Lemma 3.10 and Theorem 3.2 we get

$$
\left|\varphi_{y}(t, x)-\varphi_{y}\left(t, x^{\prime}\right)\right|
$$




$$
\begin{aligned}
& \leq \int_{0}^{t} \int_{\mathbb{R}^{d}}\left|p_{z}(t-s, x-z)-p_{z}\left(t-s, x^{\prime}-z\right)\right||q(s, z, y)| d z d s \\
& \leq c\left(A(t, x, y)+A\left(t, x^{\prime}, y\right)\right)
\end{aligned}
$$

where

$$
\begin{aligned}
A(t, x, y)= & \left|x-x^{\prime}\right|^{\gamma} \int_{0}^{t} \int_{\mathbb{R}^{d}}(t-s)^{-\gamma / \alpha}\left[\prod_{i=1}^{d} g_{t-s}\left(x_{i}-z_{i}\right)\right] \\
& \times s^{d-1}\left[\prod_{i=1}^{d} \rho_{0}^{0}\left(s, z_{i}-y_{i}\right)\right]\left[s^{\beta / \alpha}+\sum_{m=1}^{d}\left(\left|z_{m}-y_{m}\right|^{\beta} \wedge 1\right)\right] d z d s .
\end{aligned}
$$

We have

$$
\begin{aligned}
& A(t, x, y) \leq c\left|x-x^{\prime}\right|^{\gamma} \sum_{m=1}^{d}\left[\prod_{\substack{i=1 \\
i \neq m}}^{d} g_{t}\left(x_{i}-y_{i}\right)\right] \\
& \times \int_{0}^{t} \int_{\mathbb{R}} \rho_{\alpha-\gamma}^{0}\left(t-s, x_{m}-z_{m}\right) \rho_{0}^{\beta}\left(s, z_{m}-y_{m}\right) d z_{m} d s \\
& +c\left|x-x^{\prime}\right|^{\gamma}\left[\prod_{i=2}^{d} g_{t}\left(x_{i}-y_{i}\right)\right] \int_{0}^{t} \int_{\mathbb{R}} \rho_{\alpha-\gamma}^{0}\left(t-s, x_{1}-z_{1}\right) \rho_{\beta}^{0}\left(s, z_{1}-y_{1}\right) d z_{1} d s .
\end{aligned}
$$

By (2.5) we have

$$
\begin{aligned}
& \int_{0}^{t} \int_{\mathbb{R}} \rho_{\alpha-\gamma}^{0}\left(t-s, x_{m}-z_{m}\right) \rho_{0}^{\beta}\left(s, z_{m}-y_{m}\right) d z_{m} d s \\
& \leq \rho_{\alpha-\gamma+\beta}^{0}\left(t, x_{m}-y_{m}\right)+\rho_{\alpha-\gamma}^{\beta}\left(t, x_{m}-y_{m}\right)
\end{aligned}
$$

and

$$
\int_{0}^{t} \int_{\mathbb{R}} \rho_{\alpha-\gamma}^{0}\left(t-s, x_{1}-z_{1}\right) \rho_{\beta}^{0}\left(s, z_{1}-y_{1}\right) d z_{1} d s \leq \rho_{\alpha-\gamma+\beta}^{0}\left(t, x_{1}-y_{1}\right) .
$$

It follows that

$$
A(t, x, y) \leq c\left|x-x^{\prime}\right|^{\gamma} t^{-\gamma / \alpha}\left[\prod_{i=1}^{d} g_{t}\left(x_{i}-y_{i}\right)\right] .
$$

Using this, (4.30), Lemma 3.10 and (3.30) we get the assertion of Theorem 1.1 (iv).

\section{Lower bound estimates}

5.1. Lévy system. Let $\mathbb{P}^{x}, \mathbb{E}^{x}$ be the distribution and expectation for the process $X_{t}$ starting from $x \in \mathbb{R}^{d}$. By $\mathcal{F}_{t}$ we denote a natural filtration. For $x \in \mathbb{R}^{d}$ and Borel $A \subset \mathbb{R}^{d}$ we define the jumping measure

$$
J(x, A)=\mathcal{A}_{\alpha} \sum_{i=1}^{d} \int_{A} \otimes_{k \neq i} \delta_{x_{k}}\left(d y_{k}\right) a_{i i}^{\alpha}(x) \frac{d y_{i}}{\left|y_{i}-x_{i}\right|^{1+\alpha}},
$$

where $\delta_{x_{k}}$ is a Dirac measure on $\mathbb{R}$ concentrated at $x_{k}$.

The purpose of this subsection is to provide arguments for the Lévy system formula. Namely, we will show that for any $x \in \mathbb{R}^{d}$ and any non-negative measurable 
function $f$ on $\mathbb{R}_{+} \times \mathbb{R}^{d} \times \mathbb{R}^{d}$ vanishing on $\left\{(s, x, y) \in \mathbb{R}_{+} \times \mathbb{R}^{d} \times \mathbb{R}^{d} ; x=y\right\}$ and $\mathcal{F}_{t}$ stopping time $T$, we have

$$
\mathbb{E}^{x} \sum_{s \leq T} f\left(s, X_{s-}, X_{s}\right)=\mathbb{E}^{x} \int_{0}^{T} \int_{\mathbb{R}^{d}} f\left(s, X_{s}, y\right) J\left(X_{s}, d y\right) d s .
$$

Since we exactly follow the approach of Chen and Zhang (2016) we only briefly sketch the arguments.

It is well known that for $f \in C_{b}^{2}\left(\mathbb{R}^{d}\right)$,

$$
\mathcal{L} f(x)=\frac{\mathcal{A}_{\alpha}}{2} \sum_{i=1}^{d} \int_{\mathbb{R}}\left[f\left(x+a_{i i}(x) w_{i} e_{i}\right)+f\left(x-a_{i i}(x) w_{i} e_{i}\right)-2 f(x)\right] \frac{d w_{i}}{\left|w_{i}\right|^{1+\alpha}} .
$$

For $y \in \mathbb{R}^{d}$ we denote $|y|_{\infty}=\max _{i}\left\{\left|y_{i}\right|\right\}$ the sup-norm in $\mathbb{R}^{d}$. For $x \in \mathbb{R}^{d}$ and $r>0$ we denote $B(x, r)=\left\{y \in \mathbb{R}^{d},|y-x|_{\infty}<r\right\}$. Then for $f \in C_{b}^{2}\left(\mathbb{R}^{d}\right)$, we can rewrite the formula of the generator as

$$
\mathcal{L} f(x)=\lim _{r \searrow 0} \int_{B^{c}(x, r)}(f(y)-f(x)) J(x, d y) .
$$

As it has been already observed in the last section, for any function $f \in C_{b}^{2}\left(\mathbb{R}^{d}\right)$, the process

$$
M_{t}^{f}=f\left(X_{t}\right)-f\left(X_{0}\right)-\int_{0}^{t} \mathcal{L} f\left(X_{s}\right) d s
$$

is a $\left(\mathbb{P}^{x}, \mathcal{F}_{t}\right)$ martingale. Suppose that $A$ and $B$ are two bounded closed subsets of $\mathbb{R}^{d}$ having a positive distance from each other. Let $f \in C_{b}^{2}\left(\mathbb{R}^{d}\right)$ be such that $f(x)=0, x \in A$ and $f(x)=1, x \in B$. We consider a martingale transform of $M_{t}^{f}$,

$$
N_{t}^{f}=\int_{0}^{t} \mathbf{1}_{A}\left(X_{s-}\right) d M_{s}^{f}
$$

By the Ito formula, if $X_{s-} \in A$, we have

$$
d M_{s}^{f}=f\left(X_{s}\right)-f\left(X_{s-}\right)-\mathcal{L} f\left(X_{s}\right) d s=f\left(X_{s}\right)-\mathcal{L} f\left(X_{s}\right) d s .
$$

This implies that

$$
\begin{aligned}
N_{t}^{f} & =\sum_{s \leq t} \mathbf{1}_{A}\left(X_{s-}\right) f\left(X_{s}\right)-\int_{0}^{t} \mathbf{1}_{A}\left(X_{s}\right) \mathcal{L} f\left(X_{s}\right) d s \\
& =\sum_{s \leq t} \mathbf{1}_{A}\left(X_{s-}\right) f\left(X_{s}\right)-\int_{0}^{t} \mathbf{1}_{A}\left(X_{s}\right) \int f(y) J\left(X_{s}, d y\right) d s
\end{aligned}
$$

Approximating $\mathbf{1}_{B}$ by a decreasing sequence of smooth functions we show that

$$
\sum_{s \leq t} \mathbf{1}_{A}\left(X_{s-}\right) \mathbf{1}_{B}\left(X_{s}\right)-\int_{0}^{t} \mathbf{1}_{A}\left(X_{s}\right) \int_{B} J\left(X_{s}, d y\right) d s
$$

is a martingale, hence

$$
\mathbb{E}^{x} \sum_{s \leq t} \mathbf{1}_{A}\left(X_{s-}\right) \mathbf{1}_{B}\left(X_{s}\right)=\mathbb{E}^{x} \int_{0}^{t} \mathbf{1}_{A}\left(X_{s}\right) \int_{B} J\left(X_{s}, d y\right) d s .
$$


Using this and a routine measure theoretic argument, we get

$$
\mathbb{E}^{x} \sum_{s \leq t} f\left(X_{s-}, X_{s}\right)=\mathbb{E}^{x} \int_{0}^{t} \int_{\mathbb{R}^{d}} f\left(X_{s}, y\right) J\left(X_{s}, d y\right) d s
$$

for any $x \in \mathbb{R}^{d}$ and any non-negative measurable function $f$ on $\mathbb{R}^{d} \times \mathbb{R}^{d}$ vanishing on the diagonal.

Finally, following the same arguments as in Chen and Kumagai (2008, Appendix A), we obtain (5.1).

5.2. Lower bound of $p^{A}$. We essentially follow the approach from Chen and Zhang (2016), where an argument relied on certain exit and hitting times estimates was applied, but the singularity of the jumping measure forces us to use an induction argument. It is worth mentioning that such precise estimates we obtain seem possible, despite the singularity, because the projections of the jumping measure to the main axes are very regular and absolutely continuous with respect to one dimensional Lebesgue measure. Even in the case of one-dimensional Lévy processes with singular Lévy measure lower bounds for transition density may not be possible. We start with the near diagonal estimate of the transition kernel.

Lemma 5.1. For any $a>0$ there is $c=c\left(a, d, \alpha, b_{1}, b_{2}, b_{3}, \beta\right)$ and $0<t_{0} \leq 1$, $t_{0}=t_{0}\left(a, d, \alpha, b_{1}, b_{2}, b_{3}, \beta\right)$ such that for $t \leq t_{0}$ and $x, y \in \mathbb{R}^{d}$ with $|y-x|_{\infty} \leq a t^{1 / \alpha}$,

$$
p^{A}(t, x, y) \geq c t^{-d / \alpha} \text {. }
$$

Proof: By (4.27), if $|y-x|_{\infty} \leq a t^{1 / \alpha}$, we have

$$
\left|\varphi_{y}(t, x)\right| \leq c \prod_{i=1}^{d} \rho_{\alpha}^{0}\left(t, x_{i}-y_{i}\right)\left[t^{\beta / \alpha}+\sum_{m=1}^{d}\left(\left|x_{m}-y_{m}\right|^{\beta} \wedge 1\right)\right] \leq c_{1} t^{-d / \alpha} t^{\beta / \alpha} .
$$

Hence, we can find $t_{0} \leq 1$ such that for $t \leq t_{0}$ and $|y-x|_{\infty} \leq a t^{1 / \alpha}$ we have

$$
\begin{aligned}
p^{A}(t, x, y) & =p_{y}(t, x-y)+\varphi_{y}(t, x) \\
& \geq p_{y}(t, x-y)-\left|\varphi_{y}(t, x)\right| \geq c_{2} t^{-d / \alpha}-c_{1} t^{-d / \alpha} t^{\beta / \alpha} \\
& \geq c t^{-d / \alpha}
\end{aligned}
$$

Let for a Borel $D \subset \mathbb{R}^{d}$,

$$
\tau_{D}=\inf \left\{t>0 ; X_{t} \notin D\right\} \text { and } T_{D}=\inf \left\{t>0 ; X_{t} \in D\right\}
$$

be the first exit and hitting time of $D$, respectively.

Lemma 5.2. There is c such that, for $t \leq 1, R>0, x \in \mathbb{R}^{d}$,

$$
\mathbb{P}^{x}\left(\tau_{B(x, R)} \leq t\right) \leq c \frac{t}{R^{\alpha}} .
$$

Proof: Applying the strong Markow property, we obtain

$$
\begin{aligned}
& \mathbb{P}^{x}\left(\tau_{B(x, R)} \leq t\right) \\
& \leq \mathbb{P}^{x}\left(\tau_{B(x, R)} \leq t ;|X(t)-x|_{\infty} \leq R / 8\right)+\mathbb{P}^{x}\left(|X(t)-x|_{\infty} \geq R / 8\right) \\
& \leq \mathbb{P}^{x}\left(\tau_{B(x, R)} \leq t ;\left|X(t)-X\left(\tau_{B(x, R)}\right)\right|_{\infty} \geq R / 8\right) \\
& +\mathbb{P}^{x}\left(|X(t)-x|_{\infty} \geq R / 8\right)
\end{aligned}
$$




$$
\begin{aligned}
& =\mathbb{E}^{x}\left(\tau_{B(x, R)} \leq t ; P^{X\left(\tau_{B(x, R)}\right)}\left(\left|X\left(t-\tau_{B(x, R)}\right)-X\left(\tau_{B(x, R)}\right)\right|_{\infty} \geq R / 8\right)\right) \\
& +\mathbb{P}^{x}\left(|X(t)-x|_{\infty} \geq R / 8\right) \\
& \leq 2 \sup _{z} \sup _{s \leq t} \mathbb{P}^{z}\left(|X(s)-z|_{\infty} \geq R / 8\right) \\
& \leq c \frac{t}{R^{\alpha}} .
\end{aligned}
$$

The last step follows from the upper estimate (1.5) of the heat kernel $p^{A}(t, x, y)$.

Lemma 5.3. Let $r>0$ and $x, y \in \mathbb{R}^{d}$. Assume that $\left|x_{1}-y_{1}\right| \geq 6 r$ and $\max _{2 \leq i \leq d}\left|x_{i}-y_{i}\right| \leq r$. Then for $t>0$,

$$
\mathbb{P}^{x}(X(t) \in B(y, 4 r)) \geq c \frac{r t}{\left|y_{1}-x_{1}\right|^{1+\alpha}} \mathbb{P}^{x}\left(\tau_{B(x, r)} \geq t\right) \inf _{z} \mathbb{P}^{z}\left(\tau_{B(z, 2 r)}>t\right)
$$

Proof: Let $\sigma=T_{B(y, 2 r)}$ be the first hitting time. By the strong Markow property

$$
\begin{aligned}
\mathbb{P}^{x}(X(t) \in B(y, 4 r)) & \geq \mathbb{P}^{x}\left(\sigma \leq t ; \sup _{\sigma \leq s \leq \sigma+t}|X(s)-X(\sigma)|_{\infty} \leq 2 r\right) \\
& =\mathbb{E}^{x}\left(\sigma \leq t ; P^{X(\sigma)}\left(\sup _{s \leq t}|X(s)-X(\sigma)|_{\infty} \leq 2 r\right)\right) \\
& \geq \mathbb{P}^{x}(\sigma \leq t) \inf _{z} \mathbb{P}^{z}\left(\sup _{s \leq t}|X(s)-z|_{\infty} \leq 2 r\right) \\
& \geq \inf _{z} \mathbb{P}^{z}\left(\tau_{B(z, 2 r)}>t\right) \mathbb{P}^{x}\left(X\left(t \wedge \tau_{B(x, r)}\right) \in B(y, 2 r)\right) .
\end{aligned}
$$

By the Lévy system formula (5.1), we have

$$
\mathbb{P}^{x}\left(\left(X\left(t \wedge \tau_{B(x, r)}\right) \in B(y, 2 r)\right)=\mathbb{E}^{x} \int_{0}^{t \wedge \tau_{B(x, r)}} \int_{B(y, 2 r)} J\left(X_{s}, d u\right) d s .\right.
$$

We may assume $x_{1}<y_{1}$. Since $\left|x_{1}-y_{1}\right| \geq 6 r$ and $\max _{2 \leq i \leq d}\left|x_{i}-y_{i}\right| \leq r$, for $z \in B(x, r)$, we have

$$
\int_{B(y, 2 r)} J(z, d u)=\int_{y_{1}-2 r}^{y_{1}+2 r} \frac{a_{11}^{\alpha}(z) d w_{1}}{\left|w_{1}-z_{1}\right|^{1+\alpha}} \geq c \frac{r}{\left|y_{1}-x_{1}\right|^{1+\alpha}} .
$$

Hence,

$$
\begin{aligned}
\mathbb{P}^{x}\left(\left(X\left(t \wedge \tau_{B(x, r)}\right) \in B(y, 2 r)\right)\right. & \geq c \frac{r}{\left|y_{1}-x_{1}\right|^{1+\alpha}} \mathbb{E}^{x}\left[t \wedge \tau_{B(x, r)}\right] \\
& \geq c \frac{r t}{\left|y_{1}-x_{1}\right|^{1+\alpha}} \mathbb{P}^{x}\left(\tau_{B(x, r)} \geq t\right) .
\end{aligned}
$$

Lemma 5.4. There is $t_{0}>0, t_{0}=t_{0}\left(d, \alpha, b_{1}, b_{2}, b_{3}, \beta\right)$, such that for $0<t \leq t_{0}$, $x, y \in \mathbb{R}^{d}$ satisfying $\max _{2 \leq i \leq d}\left|x_{i}-y_{i}\right| \leq 2 t^{1 / \alpha}$ we have

$$
p^{A}(t, x, y) \geq c \prod_{i=1}^{d} g_{t}\left(x_{i}-y_{i}\right) .
$$

Proof: We pick $t_{0}>0$ corresponding to $a=12$ in Lemma 5.1. Due to the near diagonal estimate (5.2), it is enough to consider $\left|x_{1}-y_{1}\right| \geq 12 t^{1 / \alpha}$ and $\max _{2 \leq i \leq d}\left|x_{i}-y_{i}\right| \leq 2 t^{1 / \alpha}$. Applying Lemma 5.3 with $r=2 t^{1 / \alpha}$, we obtain

$$
p^{A}(t, x, y) \geq \int_{B(y, 4 r)} p^{A}\left(t_{1}, x, z\right) p^{A}\left(t_{2}, z, y\right) d z
$$




$$
\begin{aligned}
& \geq \inf _{z \in B(y, 4 r)} p^{A}\left(t_{2}, z, y\right) \mathbb{P}^{x}\left(\left(X\left(t_{1}\right) \in B(y, 4 r)\right)\right. \\
& \geq \quad c \inf _{z \in B(y, 4 r)} p^{A}\left(t_{2}, z, y\right) \frac{r t_{1}}{\left|y_{1}-x_{1}\right|^{1+\alpha}} \mathbb{P}^{x}\left(\tau_{B(x, r)} \geq t_{1}\right) \\
& \times \quad \inf _{z} \mathbb{P}^{z}\left(\tau_{B(z, 2 r)}>t_{1}\right),
\end{aligned}
$$

where $t_{i}>0$ with $t_{1}+t_{2}=t$.

Now, due to Lemma 5.2, we can pick $0<\lambda<1$, independently of $t$, such that

$$
\inf _{z} \mathbb{P}^{z}\left(\tau_{B(z, r)} \geq \lambda t\right) \geq 1 / 2 .
$$

Moreover, we can select $\lambda$ so small that $8 \leq 12(1-\lambda)^{1 / \alpha}$. Then for $|z-y|_{\infty} \leq 4 r=$ $8 t^{1 / \alpha} \leq 12((1-\lambda) t)^{1 / \alpha}$, by Lemma 5.1 , we have

$$
p^{A}((1-\lambda) t, z, y) \geq c t^{-d / \alpha} .
$$

Taking $t_{1}=\lambda t, t_{2}=(1-\lambda) t$ and applying (5.3) we arrive at

$$
p^{A}(t, x, y) \geq c t^{-(d-1) / \alpha} \frac{t}{\left|y_{1}-x_{1}\right|^{1+\alpha}} \geq c \prod_{i=1}^{d} g_{t}\left(x_{i}-y_{i}\right) .
$$

Proof of the lower bound estimates in Theorem 1.1 (iii): For a natural $k \leq d-1$ we define

$$
V_{k}(t)=\left\{(x, y) \in \mathbb{R}^{2 d} ; \min _{1 \leq i \leq k}\left|x_{i}-y_{i}\right| \geq t^{1 / \alpha} \text { and } \max _{k+1 \leq i \leq d}\left|x_{i}-y_{i}\right| \leq t^{1 / \alpha}\right\}
$$

We set

$$
V_{0}(t)=\left\{(x, y) \in \mathbb{R}^{2 d} ; \max _{1 \leq i \leq d}\left|x_{i}-y_{i}\right| \leq t^{1 / \alpha}\right\}
$$

and

$$
V_{d}(t)=\left\{(x, y) \in \mathbb{R}^{2 d} ; \min _{1 \leq i \leq d}\left|x_{i}-y_{i}\right| \geq t^{1 / \alpha}\right\}
$$

By a renumeration argument it is enough to prove the corresponding lower bound on $V_{k}(t), k=0, \ldots, d$. At first, we assume that $t \leq t_{0}$, where $t_{0}$ was found in Lemma 5.4. We have already proved the lower bound on $V_{0}(t)$ and $V_{1}(t)$. We show how to extend it to $V_{2}(t)$.

Thus, we consider the case $\left|x_{1}-y_{1}\right| \geq t^{1 / \alpha},\left|x_{2}-y_{2}\right| \geq t^{1 / \alpha}$ and $\max _{3 \leq i \leq d} \mid x_{i}-$ $y_{i} \mid \leq t^{1 / \alpha}$

Let $x^{\prime}=\left(y_{1}, x_{2}, \ldots, x_{d}\right)$. If $z \in B\left(x^{\prime}, t^{1 / \alpha} / 4\right)$ then $\left|x_{1}-z_{1}\right| \geq(3 / 4) t^{1 / \alpha}$, $\max _{2 \leq i \leq d}\left|x_{i}-z_{i}\right| \leq 2 t^{1 / \alpha}$ and $\left|y_{2}-z_{2}\right| \geq(3 / 4) t^{1 / \alpha}, \max _{i \neq 2}\left|y_{i}-z_{i}\right| \leq 2 t^{1 / \alpha}$. Hence, by Lemma 5.4,

$$
\begin{aligned}
& p^{A}(t, x, z) \geq c t^{-(d-1) / \alpha} \frac{t}{\left|z_{1}-x_{1}\right|^{1+\alpha}} \geq c t^{-(d-1) / \alpha} \frac{t}{\left|y_{1}-x_{1}\right|^{1+\alpha}}, \\
& p^{A}(t, z, y) \geq c t^{-(d-1) / \alpha} \frac{t}{\left|z_{2}-y_{2}\right|^{1+\alpha}} \geq c t^{-(d-1) / \alpha} \frac{t}{\left|y_{2}-x_{2}\right|^{1+\alpha}} .
\end{aligned}
$$

Finally,

$$
p^{A}(2 t, x, y) \geq \int_{B\left(x^{\prime}, t^{1 / \alpha} / 4\right)} p^{A}(t, x, z) p^{A}(t, z, y) d z
$$




$$
\begin{aligned}
& \geq c t^{-(d-1) / \alpha} \frac{t}{\left|y_{1}-x_{1}\right|^{1+\alpha}} t^{-(d-1) / \alpha} \frac{t}{\left|y_{2}-x_{2}\right|^{1+\alpha}} \int_{B\left(x^{\prime}, t^{1 / \alpha} / 4\right)} d z \\
& \geq c \frac{t}{\left|y_{1}-x_{1}\right|^{1+\alpha}} \frac{t}{\left|y_{2}-x_{2}\right|^{1+\alpha}} t^{-(d-2) / \alpha} \\
& \geq c \prod_{i=1}^{d} g_{2 t}\left(x_{i}-y_{i}\right) .
\end{aligned}
$$

This concludes the proof of the lower bound on $V_{2}(t)$. In a similar fashion, by induction argument, we show that, if $(x, y) \in V_{k}(t)$ and $t \leq t_{0}$, then

$$
\begin{aligned}
p^{A}(t, x, y) & \geq c \frac{t}{\left|y_{1}-x_{1}\right|^{1+\alpha}} \times \cdots \times \frac{t}{\left|y_{k}-x_{k}\right|^{1+\alpha}} t^{-(d-k) / \alpha} \\
& \geq c \prod_{i=1}^{d} g_{t}\left(x_{i}-y_{i}\right) \geq c p_{0}(t, x-y),
\end{aligned}
$$

which ends the proof for the case $t \leq t_{0}$. If $t>t_{0}$ then we can write $t=n t_{0}+s$, with $s<t_{0}$ and $n \in \mathbb{N}$. Then by already proved lower bound

$$
\begin{aligned}
& p^{A}(t, x, y)=\int_{\mathbb{R}^{d}} \ldots \int_{\mathbb{R}^{d}} p^{A}\left(t_{0}, x, z_{1}\right) \ldots p^{A}\left(t_{0}, z_{n}, z_{n+1}\right) p^{A}\left(s, z_{n+1}, y\right) d z_{1} \ldots d z_{n+1} \\
& \geq c^{n+1} \int_{\mathbb{R}^{d}} \ldots \int_{\mathbb{R}^{d}} p_{0}\left(t_{0}, x-z_{1}\right) \ldots p_{0}\left(t_{0}, z_{n}-z_{n+1}\right) p_{0}\left(s, z_{n+1}-y\right) d z_{1} \ldots d z_{n+1} \\
& =c^{n+1} p_{0}(t, x-y) .
\end{aligned}
$$

The proof is completed.

Proof of Theorem $1.1(v)$ : The assertion follows from Proposition 3.15 and the lower bound estimate in Theorem 1.1 (iii).

Acknowledgements. We thank the referee for useful comments and suggestions which improved the presentation of the paper.

\section{References}

R. F. Bass and Z.-Q. Chen. Systems of equations driven by stable processes. Probab. Theory Related Fields 134 (2), 175-214 (2006). MR2222382.

K. Bogdan, T. Grzywny and M. Ryznar. Density and tails of unimodal convolution semigroups. J. Funct. Anal. 266 (6), 3543-3571 (2014). MR3165234.

K. Bogdan and T. Jakubowski. Estimates of heat kernel of fractional Laplacian perturbed by gradient operators. Comm. Math. Phys. 271 (1), 179-198 (2007). MR2283957.

K. Bogdan and P. Sztonyk. Estimates of the potential kernel and Harnack's inequality for the anisotropic fractional Laplacian. Studia Math. 181 (2), 101-123 (2007). MR2320691.

K. Bogdan, P. Sztonyk and V. Knopova. Random matrices: The universality phenomenon for Wigner ensemblesheat kernel of anisotropic nonlocal operators. ArXiv Mathematics e-prints (2017). arXiv: 1704.03705.

Z.-Q. Chen and E. Hu. Heat kernel estimates for $\Delta+\Delta^{\alpha / 2}$ under gradient perturbation. Stochastic Process. Appl. 125 (7), 2603-2642 (2015). MR3332849. 
Z.-Q. Chen, P. Kim and T. Kumagai. Global heat kernel estimates for symmetric jump processes. Trans. Amer. Math. Soc. 363 (9), 5021-5055 (2011). MR2806700.

Z.-Q. Chen, P. Kim and R. Song. Dirichlet heat kernel estimates for fractional Laplacian with gradient perturbation. Ann. Probab. 40 (6), 2483-2538 (2012). MR3050510.

Z.-Q. Chen, P. Kim and R. Song. Stability of Dirichlet heat kernel estimates for non-local operators under Feynman-Kac perturbation. Trans. Amer. Math. Soc. 367 (7), 5237-5270 (2015). MR3335416.

Z.-Q. Chen and T. Kumagai. Heat kernel estimates for jump processes of mixed types on metric measure spaces. Probab. Theory Related Fields 140 (1-2), 277 317 (2008). MR2357678.

Z.-Q. Chen and X. Zhang. Heat kernels and analyticity of non-symmetric jump diffusion semigroups. Probab. Theory Related Fields 165 (1-2), 267-312 (2016). MR3500272.

A. Friedman. Partial differential equations of parabolic type. Prentice-Hall, Inc., Englewood Cliffs, N.J. (1964). MR0181836.

T. Grzywny and K. Szczypkowski. Estimates of heat kernels of non-symmetric Lévy processes. ArXiv Mathematics e-prints (2017). arXiv: 1710.07793.

T. Grzywny and K. Szczypkowski. Heat kernels of non-symmetric Lévy-type operators. ArXiv Mathematics e-prints (2018). arXiv: 1804.01313.

Y. Ishikawa. Density estimate in small time for jump processes with singular Lévy measures. Tohoku Math. J. (2) 53 (2), 183-202 (2001). MR1829978.

T. Jakubowski and K. Szczypkowski. Time-dependent gradient perturbations of fractional Laplacian. J. Evol. Equ. 10 (2), 319-339 (2010). MR2643799.

T. Jakubowski and K. Szczypkowski. Estimates of gradient perturbation series. J. Math. Anal. Appl. 389 (1), 452-460 (2012). MR2876511.

K. Kaleta and P. Sztonyk. Upper estimates of transition densities for stabledominated semigroups. J. Evol. Equ. 13 (3), 633-650 (2013). MR3089797.

K. Kaleta and P. Sztonyk. Estimates of transition densities and their derivatives for jump Lévy processes. J. Math. Anal. Appl. 431 (1), 260-282 (2015). MR3357585.

K. Kaleta and P. Sztonyk. Small-time sharp bounds for kernels of convolution semigroups. J. Anal. Math. 132, 355-394 (2017). MR3666815.

P. Kim, R. Song and Z. Vondraček. Heat kernels of non-symmetric jump processes: beyond the stable case. Potential Anal. 49 (1), 37-90 (2018). MR3817130.

V. Knopova. Compound kernel estimates for the transition probability density of a Lévy process in $\mathbb{R}^{n}$. Teor. Imovir. Mat. Stat. (89), 51-63 (2013). MR3235175.

V. Knopova and A. Kulik. Exact asymptotic for distribution densities of Lévy functionals. Electron. J. Probab. 16, no. 52, 1394-1433 (2011). MR2827465.

V. Knopova and A. Kulik. Intrinsic compound kernel estimates for the transition probability density of Lévy-type processes and their applications. Probab. Math. Statist. 37 (1), 53-100 (2017). MR3652202.

V. Knopova and A. Kulik. Parametrix construction of the transition probability density of the solution to an SDE driven by $\alpha$-stable noise. Ann. Inst. Henri Poincaré Probab. Stat. 54 (1), 100-140 (2018). MR3765882.

V. Knopova and R. L. Schilling. Transition density estimates for a class of Lévy and Lévy-type processes. J. Theoret. Probab. 25 (1), 144-170 (2012). MR2886383. 
A. N. Kochubel̆. Parabolic pseudodifferential equations, hypersingular integrals and Markov processes. Izv. Akad. Nauk SSSR Ser. Mat. 52 (5), 909-934, 1118 (1988). MR972089.

V. Kolokoltsov. Symmetric stable laws and stable-like jump-diffusions. Proc. London Math. Soc. (3) 80 (3), 725-768 (2000). MR1744782.

F. Kühn. Existence of (Markovian) solutions to martingale problems associated with Lévy-type operators. ArXiv Mathematics e-prints (2012). arXiv: 1803.05646.

F. Kühn. Lévy matters. VI, volume 2187 of Lecture Notes in Mathematics. Springer, Cham (2017). ISBN 978-3-319-60887-7; 978-3-319-60888-4. MR3701414.

F. Kühn. On martingale problems and Feller processes. Electron. J. Probab. 23, Paper No. 13, 18 (2018a). MR3771750.

F. Kühn. Solutions of Lévy-driven SDEs with unbounded coefficients as Feller processes. Proc. Amer. Math. Soc. 146 (8), 3591-3604 (2018b). MR3803683.

T. Kulczycki and M. Ryznar. Gradient estimates of harmonic functions and transition densities for Lévy processes. Trans. Amer. Math. Soc. 368 (1), 281-318 (2016). MR3413864.

A. Kulik. On weak uniqueness and distributional properties of a solution to an SDE with $\alpha$-stable noise. Stochastic Process. Appl. (2018). On-line. DOI: 10.1016/j.spa.2018.03.010.

O. A. Ladyženskaja, V. A. Solonnikov and N. N. Ural'ceva. Linear and quasilinear equations of parabolic type. Translated from the Russian by S. Smith. Translations of Mathematical Monographs, Vol. 23. American Mathematical Society, Providence, R.I. (1968). MR0241822.

E. E. Levi. Sulle equazioni lineari totalmente ellittiche alle derivate parziali. Rend. Circ. Mat. Palermo 24, 275-317 (1907).

R. Mikulevičius and C. Zhang. On the rate of convergence of weak Euler approximation for nondegenerate SDEs driven by Lévy processes. Stochastic Process. Appl. 121 (8), 1720-1748 (2011). MR2811021.

R. Mikulevičius and C. Zhang. Weak Euler approximation for Itô diffusion and jump processes. Stoch. Anal. Appl. 33 (3), 549-571 (2015). MR3339318.

A. Mimica. Heat kernel upper estimates for symmetric jump processes with small jumps of high intensity. Potential Anal. 36 (2), 203-222 (2012). MR2886459.

J. Picard. Density in small time for Levy processes. ESAIM Probab. Statist. 1, 357-389 (1995/97). MR1486930.

J. Picard. On the existence of smooth densities for jump processes. Probab. Theory Related Fields 105 (4), 481-511 (1996). MR1402654.

J. Picard. Density in small time at accessible points for jump processes. Stochastic Process. Appl. 67 (2), 251-279 (1997). MR1449834.

E. Priola, A. Shirikyan, L. Xu and J. Zabczyk. Exponential ergodicity and regularity for equations with Lévy noise. Stochastic Process. Appl. 122 (1), 106-133 (2012). MR2860444.

E. Priola and J. Zabczyk. Structural properties of semilinear SPDEs driven by cylindrical stable processes. Probab. Theory Related Fields 149 (1-2), 97-137 (2011). MR2773026.

R. L. Schilling, P. Sztonyk and J. Wang. Coupling property and gradient estimates of Lévy processes via the symbol. Bernoulli 18 (4), 1128-1149 (2012). MR2995789. 
X. Sun and Y. Xie. Ergodicity of stochastic dissipative equations driven by $\alpha$-stable process. Stoch. Anal. Appl. 32 (1), 61-76 (2014). MR3175815.

P. Sztonyk. Approximation of stable-dominated semigroups. Potential Anal. 33 (3), 211-226 (2010a). MR2718253.

P. Sztonyk. Regularity of harmonic functions for anisotropic fractional Laplacians. Math. Nachr. 283 (2), 289-311 (2010b). MR2604123.

P. Sztonyk. Estimates of densities for Lévy processes with lower intensity of large jumps. Math. Nachr. 290 (1), 120-141 (2017). MR3604626.

L. Wang and X. Zhang. Harnack inequalities for SDEs driven by cylindrical $\alpha$-stable processes. Potential Anal. 42 (3), 657-669 (2015). MR3336993.

L. Xie and X. Zhang. Heat kernel estimates for critical fractional diffusion operators. Studia Math. 224 (3), 221-263 (2014). MR3294616.

$\mathrm{X}$. Zhang. Derivative formulas and gradient estimates for SDEs driven by $\alpha$-stable processes. Stochastic Process. Appl. 123 (4), 1213-1228 (2013). MR3016221. 